\title{
Continuity of the bending map
}

\author{
CYRIL LECUIRE
}

May 2004

\begin{abstract}
The bending map of a hyperbolic 3-manifold maps a convex cocompact hyperbolic metric on a hyperbolic 3-manifold with boundary to its bending measured geodesic lamination. As proved in $[\mathrm{KeS}]$ and $[\mathrm{KaT}]$, this map is continuous. In the present paper we study the extension of this map to the space of geometrically finite hyperbolic metrics. We introduce a relationship on the space of measured geodesic laminations and shows that the quotient map obtained from the bending map is continuous.
\end{abstract}

\section{Introduction}

Let $M$ be a compact, orientable 3-manifold with boundary. Assume that $M$ is hyperbolic, namely that the interior of $M$ is endowed with a complete metric $\sigma$ of constant sectional curvature -1 . Assume also that $\partial M$ contains a surface with genus greater than 1. A fundamental subset of $(M, \sigma)$ is its convex core $N(\sigma)$. This core $N(\sigma)$ is defined as the smallest non-empty closed subset of the interior of $M$ which is locally convex and homotopically equivalent to $M$. Its boundary $\partial N(\sigma)$ endowed with the intrinsic metric (given by rectifiable path length) is isometric to a hyperbolic surface of finite volume and can be embedded in a natural way into $\partial M$. This surface is bent along a geodesic lamination and the amount of bending is described by a measured geodesic lamination called the bending measured geodesic lamination of $\sigma$ (cf. [Th] or [CEG]). This yields a bending map $b$ which to a complete hyperbolic metric associates its bending measured geodesic lamination.

In [Bo2], F. Bonahon considers quasi-isometric deformations of a given metric $\sigma$ on $\operatorname{int}(M)$, namely hyperbolic metrics $\sigma^{\prime}$ on $\operatorname{int}(M)$ for which there exists a diffeomorphism $(\operatorname{int}(M), \sigma) \rightarrow\left(\operatorname{int}(M), \sigma^{\prime}\right)$ whose differential is uniformly bounded. Let $\mathscr{Q} \mathscr{D}(\sigma)$ be the space of quasi-isometric deformations of a given metric $\sigma$, where we identify two deformations $(\operatorname{int}(M), \sigma) \rightarrow\left(\operatorname{int}(M), \sigma^{\prime}\right)$ and $(\operatorname{int}(M), \sigma) \rightarrow\left(\operatorname{int}(M), \sigma^{\prime \prime}\right)$ if they are isotopic. The continuity of the bending map $b: \mathscr{Q} \mathscr{D}(\sigma) \rightarrow \mathscr{M} \mathscr{L}(\partial M)$ is proved in [KaT, using ideas of Thurston. Its differentiability (in a weak sense) is proved in Bo2].

A complete hyperbolic metric $\sigma$ on $\operatorname{int}(M)$ is said to be convex cocompact if $N(\sigma)$ is compact. If $\sigma$ is convex cocompact, then $\mathscr{Q} \mathscr{D}(\sigma)$ is the set of convex cocompact metrics on $\operatorname{int}(M)$. In this case, the continuity of $b: \mathscr{Q} \mathscr{D}(\sigma) \rightarrow \mathscr{M} \mathscr{L}(\partial M)$ has been proved in [KeS] and its image has been described in [BoO] and [Le1]. A complete hyperbolic metric $\sigma$ on int $(M)$ is said to be geometrically finite if $N(\sigma)$ has finite volume. When $\sigma$ is a geometrically finite metric, $\mathscr{Q} \mathscr{D}(\sigma)$ is the set of geometrically finite having the same parabolic subgroups as $\sigma$. In the present paper, we are addressing the question of the continuity of the bending map on the whole set of geometrically finite metrics. In particular, we are interested in sequence converging to a limit with some new parabolics.

Since we want to consider metric which do not have the same parabolic subgroups, we have to allow deformations which are not quasi-isometric. We will consider the set isotopy classes of hyperbolic metric on the interior of $M$. Two metrics $\sigma_{1}$ and $\sigma_{2}$ are identified if there exists a diffeomorphism $f: M \rightarrow M$ isotopic to the identity such that $\sigma_{1}=f^{*} \sigma_{2}$. We will consider the set $\mathscr{G} \mathscr{F}(M)$ of isotopy classes of geometrically finite hyperbolic metrics which are not fuchsian. We will topologize $\mathscr{G} \mathscr{F}(M)$ in the following way. Let us choose a point $x$ in $\operatorname{int}(M)$. A metric $\sigma_{2}$ lies in a $(k, r)$-neighborhood of $\sigma_{1}$ if there exists a diffeomorphism $g: M \rightarrow M$ isotopic to the 
identity such that the restriction of $g$ to the ball $B(x, r) \subset\left(M, \sigma_{1}\right)$ is a $k$-quasi-isometry into its image in $\left(M, \sigma_{2}\right)$. We obtain a basis of neighborhoods of $\sigma_{1}$ by letting $k$ tend to 1 and $r$ tend to $+\infty$. The topology defined in this way does not depend on the choice of the point $x$. For a metric $\sigma \in \mathscr{G} \mathscr{F}(M), \mathscr{Q} \mathscr{F}(\sigma)$ can be viewed as a subset of $\mathscr{G} \mathscr{F}(M)$. The topology of $\mathscr{Q} \mathscr{F}(\sigma)$ considered as a subset of $\mathscr{G} \mathscr{F}(M)$ coincides with the topology given by quasi-isometric deformations

The bending map $b_{\mathscr{G} \mathscr{F}}(M): \mathscr{G} \mathscr{F}(M) \rightarrow \mathscr{M} \mathscr{L}(\partial M)$ maps an isotopy class of geometrically finite metrics to its bending measured geodesic lamination. The image of $b_{\mathscr{G}} \mathscr{F}$ has been described in [BoO] and [Le1], it is the set $\mathscr{P}(M)$ of measured geodesic laminations satisfying the following conditions:

- a) no closed leaf of $\lambda$ has a weight greater than $\pi$;

- b) $\exists \eta>0$ such that, for any essential annulus $E, i(\partial E, \lambda) \geq \eta$;

- c) $i(\lambda, \partial D)>2 \pi$ for any essential disc $D$.

Taking a careful look at the behavior of the map $b \mathscr{G} \mathscr{F}$, we notice that it is not a continuous map. If a metric $\sigma$ lies in $\mathscr{G} \mathscr{F}(M)$ but has some rank one cusps, its measured geodesic laminations $\lambda$ has some compact leaves with a weight equal to $\pi$. Let us denote by $\lambda^{(p)}$ the union of the leaves of $\lambda$ which have a weight equal to $\pi$. Using the result of $\mathrm{BoO}$, it is not hard to construct a sequence of metrics $\sigma_{n} \in \mathscr{G} \mathscr{F}(M)$ with measured geodesic laminations $\lambda_{n}$ such that the sequence $\left(\lambda_{n}\right)$ converges to a measured geodesic lamination $\lambda_{\infty}$ which differs from $\lambda$ only on $\lambda^{(p)}$ and which have some leaves with a weight greater than $\pi$. Using some arguments of [Le1], we get that a subsequence of $\left(\sigma_{n}\right)$ converges to a geometrically finite metric $\sigma_{\infty}$. Since $\lambda_{\infty}$ does not satisfies condition $a$ ), it is not the bending measured geodesic lamination of $\sigma_{\infty}$. Thus we get that $b_{\mathscr{G} \mathscr{F}}$ is not continuous on any neighborhood of a metric with some rank one cusps. To overcome this difficulty, we will quotient the space $\mathscr{M} \mathscr{L}(\partial M)$ of measured geodesic laminations by the following relationship :

Let $\lambda, \mu \in \mathscr{M} \mathscr{L}(\partial M)$ be two measured geodesic laminations and let us denote by $\lambda^{\prime}$ (resp. $\left.\mu^{\prime}\right)$ the measured geodesic laminations obtained by replacing by $\pi$ the weights of the leaves of $\lambda$ (resp. $\mu$ ) which have a weight greater than $\pi$; we set $\lambda \mathscr{R} \mu$ if and only if $\lambda^{\prime}=\mu^{\prime}$. We denote by $\dot{\lambda}$ the class of $\lambda$ modulo $\mathscr{R}$.

Let us endow $\mathscr{M} \mathscr{L}(\partial M)$ with the weak* topology and $\mathscr{M} \mathscr{L}(\partial M) / \mathscr{R}$ with the quotient topology. From $b_{\mathscr{G} \mathscr{F}}(M)$ we obtain a quotient map $b_{\mathscr{R}}: \mathscr{G} \mathscr{F}(M) \rightarrow \mathscr{P}^{+}(M) / \mathscr{R}$. We will prove the following result :

Theorem 1. The map $b_{\mathscr{R}}$ from $\mathscr{G} \mathscr{F}(M)$ to $\mathscr{M} \mathscr{L}(\partial M) / \mathscr{R}$ is a continuous map.

In [Le2], we show the reverse of this theorem. This gives rise to a criterion for the strong convergence of a sequence of geometrically finite representations $\rho_{n} \in U(M)$.

The paper is organized as follows. In section 1, we state some definitions and we prove some facts about $\mathscr{M} \mathscr{L}(\partial M) / \mathscr{R}$. In section 2 , we study convex pleated surfaces and prove the continuity of the bending measured geodesic laminations of a converging sequence of convex pleated surfaces. In section 3 we use the results of section 2 to prove the continuity of $b_{\mathscr{R}}$.

\section{Definitions}

Let $\sigma$ be a hyperbolic metric (up to isotopy) on $\operatorname{int}(M)$. Given an isometry from the interior of $\tilde{M}$ to $\mathbb{H}^{3}$, the covering transformations yield a discrete faithful representation 
$\rho: \pi_{1}(M) \rightarrow \operatorname{Isom}\left(\mathbb{H}^{3}\right)$. The representations that appear in this way will be called representations associated to $\sigma$. The set of representations associated to $\sigma$ is the set of all representations conjugated to $\rho$. The image $\rho\left(\pi_{1}(M)\right)$ is a finitely generated torsion free Kleinian group and $\operatorname{int}(M)$ endowed with $\sigma$ is isometric to $\mathbb{H}^{3} / \rho\left(\pi_{1}(M)\right)$. The Nielsen core of $\mathbb{H}^{3} / \rho\left(\pi_{1}(M)\right)$ is the quotient by $\rho\left(\pi_{1}(M)\right)$ of the convex hull $C(\rho)$ of the limit set $L_{\rho}$ of $\rho\left(\pi_{1}(M)\right)$ (see [Th, chap 8] for details). This set $N(\rho)$ and the convex core defined in the introduction are isometric and from now on we will identify them. The thick part, $N(\rho)^{e p}$ of the Nielsen core is the complementary of the cuspidal part of $\mathbb{H}^{3} / \rho\left(\pi_{1}(M)\right)$ in $N(\rho)$. The representation $\rho$ is geometrically finite when $N(\rho)^{e p}$ is compact (here it is equivalent to say that $N(\rho)$ has finite volume) and convex cocompact when $N(\rho)$ is compact.

When $\rho$ is geometrically finite and not fuchsian, the natural retraction from $\mathbb{H}^{3} / \rho\left(\pi_{1}(M)\right)$ to $N(\rho)$ associates to $\sigma$ a homeomorphism (defined up to isotopy) $h: M \rightarrow N(\rho)^{e p}$. Such a homeomorphism will be said to be associated to $\sigma$.

Let $\sigma \in \mathscr{C} \mathscr{C}(M)$ and let $\rho$ and $h$ be respectively a representation and a homeomorphism associated to $\sigma$. Let $\tilde{h}: \tilde{M} \rightarrow \mathbb{H}^{3}$ be a lift of $h$, we will define the compactification $\bar{M}$ of $\tilde{M}$ as being the closure of $\tilde{h}(\tilde{M})$ in the usual compactification of $\mathbb{H}^{3}$ by the unit ball.

Let $\left(\sigma_{n}\right)$ be a sequence of isotopy classes of complete hyperbolic metrics on the interior of $M$. The sequence $\left(\sigma_{n}\right)$ converges algebraically when there is a sequence of representations $\rho_{n}: \pi_{1}(M) \rightarrow \operatorname{Isom}\left(\mathbb{H}^{3}\right)$ associated to $\sigma_{n}$ (as above) that converges algebraically; namely $\rho_{n}(g)$ converges for any $g \in \pi_{1}(M)$. We obtain a new representation $\rho_{\infty}: \pi_{1}(M) \rightarrow \operatorname{Isom}\left(\mathbb{H}^{3}\right)$ defined by $\rho_{\infty}(g)=\lim _{n \longrightarrow \infty} \rho_{n}(g)$ for any $g \in \pi_{1}(M)$. This representation $\rho_{\infty}$ is discrete and faithful (cf. [Jor]). This representation defines a metric on a manifold homotopy equivalent to $M$. This manifold might not be homeomorphic to $M$ (examples are given in [AnC]).

The sequence $\left(\sigma_{n}\right)$ converges geometrically if there is a sequence of representations $\rho_{n}: \pi_{1}(M) \rightarrow I \operatorname{som}\left(\mathbb{H}^{3}\right)$ associated to the $\sigma_{n}$ such that $\left(\rho_{n}\left(\pi_{1}(M)\right)\right.$ converges geometrically. The sequence of groups $\left(\rho_{n}\left(\pi_{1}(M)\right)\right.$ converges geometrically to a group $\Gamma_{\infty} \subset \operatorname{Isom}\left(\mathbb{H}^{3}\right)$ if and only if :

- for any sequence $a_{n} \in \rho_{n}\left(\pi_{1}(M)\right)$, any accumulation point $a_{\infty}$ of $\left\{a_{n} \mid n \in \mathbb{N}\right\}$ lies in $\Gamma_{\infty}$;

- any element $a_{\infty}$ of $\Gamma_{\infty}$ is the limit of a sequence $a_{n} \in \Gamma_{n}$.

The sequence $\rho_{n}\left(\pi_{1}(M)\right)$ converges strongly if there is a sequence of representations $\rho_{n}$ associated to $\sigma_{n}$ such that $\left(\rho_{n}\right)$ converges algebraically to a representation $\rho_{\infty}$ and that $\left(\rho_{n}\left(\pi_{1}(M)\right)\right)$ converges geometrically to $\rho_{\infty}\left(\pi_{1}(M)\right)$. If $\left(\sigma_{n}\right)$ converges to $\sigma$ for the topology defined in the introduction, then $\left(\sigma_{n}\right)$ converges strongly to $\sigma_{\infty}$ (cf. [CEG]).

\section{Geodesic laminations and the relationship $\mathscr{R}$}

A geodesic lamination $L$ on $\mathbb{H}^{2}$ is a closed subset which is the disjoint union of complete geodesics. A complete geodesic lying in $L$ is a leaf of $L$.

A measured geodesic lamination $\lambda$ is a transverse measure for some geodesic lamination $|\lambda|$. Any $\operatorname{arc} k \approx[0,1]$ embedded in $S$ transversely to $|\lambda|$, such that $\partial k \subset S-\lambda$, is endowed with an additive measure $d \lambda$ such that :

- the support of $d \lambda_{\mid k}$ is $|\lambda| \cap k$;

- if an arc $k$ can be homotoped into $k^{\prime}$ by a homotopy respecting $|\lambda|$ then $\int_{k} d \lambda=\int_{k^{\prime}} d \lambda$. We will denote by $\mathscr{M} \mathscr{L}\left(\mathbb{H}^{2}\right)$ the space of measured geodesic lamination topologised with the topology of the weak* convergence.

Let $S$ be a surface (which may not be compact) endowed with a complete hyperbolic metric with finite area. A geodesic lamination in $S$ is a compact subset which is the disjoint union of simple complete geodesics. Using the fact that two complete hyperbolic metrics with finite area 
on $S$ are quasi-isometric, this definition can be made independent of the chosen metric on $S$ (see for example [t1]). A measured geodesic lamination is a transverse measure for some geodesic lamination as defined above. Let $\gamma$ be a weighted simple closed geodesic with support $|\gamma|$ and weight $w$ and let $\lambda$ be a measured geodesic lamination. The intersection number of $\gamma$ and $\lambda$ is defined by $i(\gamma, \lambda)=w \int_{|\gamma|} d \lambda$. The weighted simple closed curves are dense in $\mathscr{M} \mathscr{L}(S)$ and this intersection number extends continuously to a function $i: \mathscr{M} \mathscr{L}(S) \times \mathscr{M} \mathscr{L}(S) \rightarrow \mathbb{R}$ (cf. [Bo1]).

A measured geodesic lamination $\lambda \in \mathscr{M} \mathscr{L}(S)$ is arational if for any simple closed curve $c$ which is not homotopic to a cusp, $i(c, \lambda)=\int_{c} d \lambda>0$.

Let us recall the definition of $\mathscr{R}$ given in the introduction.

Let $\lambda, \mu \in \mathscr{M} \mathscr{L}(S)$ be two measured geodesic laminations. Let us denote by $\lambda^{\prime}$ (resp. $\mu^{\prime}$ ) the measured geodesic lamination obtained by replacing by $\pi$ the weights of the compact leaves of $\lambda$ (resp. $\mu$ ) which have a weight greater than $\pi$. We will say that $\lambda$ is related to $\mu$ by the relationship $\mathscr{R}$, and we will write $\lambda \mathscr{R} \mu$, if and only if $\lambda^{\prime}=\mu^{\prime}$. We will denote by $\dot{\lambda}$ the class of $\lambda$ modulo $\mathscr{R}$ and we will topologise $\mathscr{M} \mathscr{L}(S)$ with the quotient topology of the weak* topology on $\mathscr{M} \mathscr{L}(S)$.

Let $\partial_{\chi<0} M$ be the union of the connected components of $\partial M$ with negative Euler characteristic. To simplify the notations, we will note $\mathscr{M} \mathscr{L}(\partial M)$ for $\mathscr{M} \mathscr{L}\left(\partial_{\chi<0} M\right)$.

Let $\left(\lambda_{n}\right)$ be a sequence of measured geodesic laminations such that $\left(\dot{\lambda}_{n}\right)$ converges to $\dot{\lambda}$ in $\mathscr{M} \mathscr{L}(\partial M) / \mathscr{R}$ and let $\lambda \in \mathscr{M} \mathscr{L}(\partial M)$ a representative of $\dot{\lambda}$. Let us denote by $\lambda^{(p)}$ the union of the compact leaves of $\lambda$ with a weight greater than $\pi$. We have the following:

Claim 2.1. Let $\lambda \in \mathscr{M} \mathscr{L}(\partial M)$ and let $\lambda^{\prime}$ be the representative of $\dot{\lambda}$ whose compact leaves have all a weight less than or equal to $\pi$. If $k \subset \partial M$ is a simple arc such that the points of $k \cap|\dot{\lambda}|$ are transverse intersections, then we have $\int_{k} d \lambda^{\prime} \leq \underline{\lim } \int_{k} d \lambda_{n}$. Furthermore, if $k$ does not intersect a leaf of $\lambda^{\prime}$ with a weight equal to $\pi$, then $\int_{k} d \lambda_{n}$ converges to $\int_{k} d \lambda$.

Proof. Let $k \subset \partial M$ be a simple arc such that the points of $k \cap|\dot{\lambda}|$ are transverse intersections. The set $\mathscr{V}_{k, \varepsilon}(\lambda)=\left\{\gamma \in \mathscr{M} \mathscr{L}(\partial M) /\left|\int_{k} d \gamma-\int_{k} d \lambda\right|<\varepsilon\right\}$ is a neighborhood of $\lambda$ in $\mathscr{M} \mathscr{L}(\partial M)$. Since $\dot{\lambda}_{n}$ converges to $\dot{\lambda}$, for any $\varepsilon$, there is $n_{\varepsilon}$ such that for any $n \geq n_{\varepsilon}, \exists \alpha_{n}$ with $\alpha_{n} \mathscr{R} \lambda_{n}$ and $\alpha_{n} \in \mathscr{V}_{k, \varepsilon}(\lambda)$. So, for $n \geq n_{\varepsilon}$, we have $\left|\int_{k} d \alpha_{n}-\int_{k} d \lambda_{n}\right|<\varepsilon$. We have $\int_{k} d \lambda^{\prime} \leq \int_{k} d \alpha$ for any $\alpha \in \mathscr{M} \mathscr{L}(\partial \mathscr{M})$ satisfying $\alpha \mathscr{R} \lambda$. So we get $\int_{k} d \lambda^{\prime} \leq \underline{\lim } \int_{k} d \lambda_{n}$.

If $k$ does not intersect any leaf of $\lambda^{\prime}$ with a weight equal to $\pi$, then we have $\int_{k} d \alpha=\int_{k} d \lambda$ for any $\alpha$ such that $\alpha \mathscr{R} \lambda$. So we have $\varepsilon>\left|\int_{k} d \alpha_{n}-\int_{k} d \lambda\right|=\left|\int_{k} d \lambda_{n}-\int_{k} d \lambda\right|$ for any $n \geq n_{\varepsilon}$. Letting $n$ tend to $\infty$, we get that $\int_{k} d \lambda_{n}$ converges to $\int_{k} d \lambda$.

Let $\lambda, \mu \in \mathscr{M} \mathscr{L}(\partial M)$ be two measured geodesic laminations. If $\lambda \mathscr{R} \mu$, then $\lambda$ and $\mu$ share the same support. Thus we can define the support $|\dot{\lambda}|$ of an element $\dot{\lambda}$ of $\mathscr{M} \mathscr{L}(\partial M)$ as being the support of any representative of $\dot{\lambda}$.

Claim 2.2. Let $\left(\lambda_{n}\right) \in \mathscr{M} \mathscr{L}(\partial M)$ be a sequence of measured geodesic laminations such that $\left(\dot{\lambda}_{n}\right)$ converges to $\dot{\lambda}$ and that $\left|\lambda_{n}\right|$ converges to some geodesic lamination $L$ in the Hausdorff topology. We have $|\dot{\lambda}| \subset L$.

Proof. Let $x$ be a point of $|\dot{\lambda}|$, let $\varepsilon>0$ be a real number and let $k$ be a geodesic arc intersecting $|\dot{\lambda}|$ transversely, with length $\varepsilon$ such that $x$ lies in the interior of $k$. Since $\int_{k} d \lambda^{\prime}>0$, we deduce from 2.1 that, for $n$ large enough, we have $\int_{k} d \lambda_{n} \geq \frac{\int_{k} d \lambda^{\prime}}{2}>0$. Therefore $k$ intersects $\lambda_{n}$ and $x$ lies in an $\varepsilon$-neighborhood of $\left|\lambda_{n}\right|$. Considering a covering of $|\dot{\lambda}|$ by discs with radius $\varepsilon$ and with centers lying in $|\dot{\lambda}|$, we get that, for $n$ large enough, $|\dot{\lambda}|$ lies in an $\varepsilon$-neighborhood of $\left|\lambda_{n}\right|$. Letting $\varepsilon$ tend to 0 , we get $|\dot{\lambda}| \subset L$. 
Claim 2.1 can also be used to prove that the space $\mathscr{M} \mathscr{L}(\partial M) / \mathscr{R}$ is a Hausdorff space.

Lemma 2.3. The space $\mathscr{M} \mathscr{L}(\partial M) / \mathscr{R}$ is a Hausdorff space.

Proof. Let $\dot{\lambda}$ and $\dot{\mu}$ be two elements of $\mathscr{M} \mathscr{L}(S) / \mathscr{R}$ such that any neighborhood of $\dot{\lambda}$ intersects any neighborhood of $\dot{\alpha}$. So there is a sequence of measured geodesic laminations $\lambda_{n}$ such that $\dot{\lambda}_{n}$ converges simultaneously to $\dot{\lambda}$ and to $\dot{\mu}$. Let us denote by $\lambda^{(p)}$ (resp. $\mu^{(p)}$ ) the union of the compact leaves of $\lambda$ (resp. $\mu$ ) with a weight greater than $\pi$. Let $\lambda^{\prime}$ (resp. $\mu^{\prime}$ ) be the representative of $\dot{\lambda}$ (resp. $\dot{\mu}$ ) whose compact leaves have all a weight less than or equal to $\pi$. Let $k \subset \partial M-\lambda^{(p)}$ be a simple arc intersecting $|\lambda|$ and $|\mu|$ transversely so that $\int_{k} d \lambda<\pi$. By Claim [2.1] we have $\int_{k} d \mu^{\prime}=\lim _{n \longrightarrow+\infty} \int_{k} d \lambda_{n}<\pi$. It follows that $\mu^{(p)} \subset \lambda^{(p)}$. Reversing the roles of $\lambda$ and of $\mu$, we get $\mu^{(p)}=\lambda^{(p)}$. It follows also that we have the equality $\int_{k} d \mu=\int_{k} d \lambda$ for any arc $k \subset \partial M-\lambda^{(p)}$. This yields the conclusion $\dot{\lambda}=\dot{\mu}$.

The following variation of Claim 2.1 will be used in the present paper.

Claim 2.4. If $c$ is a simple compact curve that does not intersect $\lambda^{(p)}$ transversely, then the sequence $i\left(c, \lambda_{n}\right)$ converges to $i(c, \lambda)$.

Proof. If the points of $c \cap|\dot{\lambda}|$ are transverse intersections, we get the conclusion by cutting $c$ into two arcs and by applying Claim 2.1 to these two arcs.

Let us now consider the case where $c \subset|\dot{\lambda}|$. We will show that any subsequence of $\left(\lambda_{n}\right)$ contains a subsequence such that $i\left(c, \lambda_{n}\right)$ converges to $i(c, \lambda)=0$. Up to extracting a subsequence, we can assume that $\left|\lambda_{n}\right|$ converges in the Hausdorff topology. Let $\tau$ be a train track carrying all the $\lambda_{n}$ for $n$ large enough (see [Bo1] and [Ot1 for details about train tracks). By Claim 2.2. $\lambda$ is carried by a subtrack of $\tau$. Let $b_{1}, b_{2}, \ldots, b_{p}$ be the branches of $\tau$. Given a measured geodesic lamination $\alpha$ carried by a subtrack of $\tau$, the measure $b_{i}(\alpha)$ of a tie of $b_{i}$ does not depend on the choice of the tie. By Claim 2.1 if $b_{i}$ is a branch of $\tau$ not carrying a leaf of $\lambda^{(p)}$, then $b_{i}\left(\lambda_{n}\right) \rightarrow b_{i}(\lambda)$. Since $c$ is a leaf of $\lambda$, it is carried by a subtrack $\tau_{c}$ of $\tau$. For $n$ large enough, we have $i\left(c, \lambda_{n}\right)=\frac{1}{2} \sum_{j \in J} b_{j}\left(\lambda_{n}\right)$ where $\left\{b_{j} \mid j \in J\right\}$ is the set of branches of $\tau-\tau_{c}$ intersecting $\tau_{c}$. Such a branch $b_{j}$ does not carry a leaf of $\lambda^{(p)}$. It follows then from Claim 2.1 that $b_{j}\left(\lambda_{n}\right)$ converges to $b_{j}(\lambda)=0$. Finally, we have $i\left(c, \lambda_{n}\right) \longrightarrow 0=i(c, \lambda)$.

\section{Convex pleated surfaces}

A pleated surface in a complete hyperbolic 3-manifold $M$ is a map $f: S \rightarrow M$ from a surface $S$ to $M$ with the following properties:

- the path metric obtained by pulling back the hyperbolic metric of $M$ by $f$ is a hyperbolic metric metric $s$ on $S$;

- every point of $S$ lies in the interior of some $s$-geodesic arc which is mapped into a geodesic $\operatorname{arc}$ in $M$;

- if $c \in S$ is a simple closed curve lying in a cusp of $S$ and if $c$ does not bound a disc in $S$, then $f(c)$ does not bound a disc in $M$.

A map $\hat{f}: \mathbb{H}^{2} \rightarrow \mathbb{H}^{3}$ is a pleated map if any point of $\mathbb{H}^{2}$ lies in the interior of a geodesic arc which is mapped by $\hat{f}$ into a geodesic arc.

The pleating locus of a pleated map is the set of points of $\mathbb{H}^{2}$ where the map fails to be an isometry. The pleating locus of a pleated map is a geodesic lamination (cf. [Th]). 
An abstract pleated surface is a triple $(\hat{f}, \Gamma, \rho)$ where $\hat{f}: \mathbb{H}^{2} \rightarrow \mathbb{H}^{3}$ is a pleated map, $\Gamma$ is a lattice in $\operatorname{Isom}\left(\mathbb{H}^{2}\right)$ and $\rho: \Gamma \rightarrow \operatorname{Isom}\left(\mathbb{H}^{3}\right)$ is a discrete representation (which may not be faithful) such that for any $a \in \Gamma$, we have $\hat{f} \circ a=\rho(a) \circ \hat{f}$ and that if $a \in \Gamma$ is a parabolic isometry then $\rho(a)$ is also a parabolic isometry.

Abstract pleated surfaces and pleated surfaces are related as follows:

When $\rho(\Gamma)$ has no torsion, the abstract pleated surface $(\hat{f}, \Gamma, \rho)$ induces a pleated surface $f: S \rightarrow M$ where $S \approx \mathbb{H}^{2} / \Gamma, M \approx \mathbb{H}^{3} / \rho(\Gamma)$ and where $f$ is the quotient map from $\hat{f}$.

If $f: S \rightarrow M$ is a pleated surface, consider isometric covering maps $\mathbb{H}^{2} \rightarrow S$ and $\mathbb{H}^{3} \rightarrow M$. These maps yield representations $r: \pi_{1}(S) \rightarrow \operatorname{Isom}\left(\mathbb{H}^{2}\right)$ and $R: \pi_{1}(M) \rightarrow \operatorname{Isom}\left(\mathbb{H}^{3}\right)$ and by lifting $f$ to a map $\hat{f}: \mathbb{H}^{2} \rightarrow \mathbb{H}^{3}$ we get an abstract pleated surface $\left(\hat{f}, r\left(\pi_{1}(S)\right), R \circ f_{*}\right)$.

In the following we will omit the adjective abstract and assume that our (abstract) pleated surfaces are torsion free.

We will consider the following topology on the space of abstract pleated surfaces. A sequence $\left(\hat{f}_{n}, \Gamma_{n}, \rho_{n}\right)$ of pleated surfaces converge to a pleated surface $(\hat{f}, \Gamma, \rho)$ if and only if :

- $\left(\Gamma_{n}\right)$ converges geometrically to $\Gamma$;

- for any sequence $a_{n} \in \Gamma_{n}$ converging to $a \in \Gamma, \rho_{n}\left(a_{n}\right)$ converges to $\rho(a)$;

- $\hat{f}_{n}$ converges to $\hat{f}$ on any compact set of $\mathbb{H}^{2}$.

Let $(\hat{f}, \Gamma, \rho)$ be a (abstract) pleated surface (without torsion), let $L \subset \mathbb{H}^{2}$ be the pleating locus of $\hat{f}$ and let $P$ be a connected component of $\mathbb{H}^{2}-L$. The surface $\hat{f}(P)$ lies in a geodesic plane $\Pi_{P}$. Given an orientation of $\mathbb{H}^{2}, \Pi_{P}$ inherits a natural orientation and we denote by $H_{P}^{+}$ (resp. $H_{P}^{-}$) the half-space bounded by $\Pi_{P}$ such that the union of a direct frame of $\Pi_{P}$ and of the inward normal vector to $\Pi_{P}$ is a direct frame of $\mathbb{H}^{3}$ (resp. indirect).

A pleated surface $(\hat{f}, \Gamma, \rho)$ with pleating locus $L$ is a convex pleated surface if :

1) there is $\epsilon \in\{+,-\}$ such that for any component $P$ of $\mathbb{H}^{2}-L, \hat{f}\left(\mathbb{H}^{2}\right)$ lies in $H_{P}^{\epsilon}$;

2) the interior of $C_{\hat{f}}=\bigcap\left\{H_{P_{i}}^{\epsilon} / P_{i}\right.$ is a connected component of $\left.\mathbb{H}^{2}-\hat{L}\right\}$ is not empty.

If a pleated surface $(\hat{f}, \Gamma, \rho)$ satisfies 1$)$ but not 2$)$, namely if $C_{\hat{f}}$ as empty interior, we will call it an even pleated surface.

In the following, for any convex pleated surface $(\hat{f}, \Gamma, \rho)$, we will choose the orientation of $\mathbb{H}^{2}$ so that for any component $P$ of $\mathbb{H}^{2}-L, \hat{f}\left(\mathbb{H}^{2}\right)$ lies in $H_{P}^{+}$.

Lemma 3.1. The set of convex and even pleated surfaces is a closed subset of the set of pleated surfaces.

Proof. Let $\left(\hat{f}_{n}, \Gamma_{n}, \rho_{n}\right)$ be a sequence of convex and even pleated surfaces converging to $\left(\hat{f}_{\infty}, \Gamma_{\infty}, \rho_{\infty}\right)$. Let us follow $\mathrm{BoO}$. Lemmas 20 and 21], to show that $\hat{f} \infty$ is either a convex pleated surface or an even pleated surface. Let us denote by $\hat{L}_{n}$ the pleating locus of $\left(\hat{f}_{n}, \Gamma_{n}, \rho_{n}\right)$ and let us consider a geodesic lamination $\hat{L}_{\infty}$ lying in the closure of $\left\{\hat{L}_{n} / n \in \mathbb{N}\right\}$ in the Hausdorff topology. By CEG the pleating locus of $\hat{f}_{\infty}$ lies in $\hat{L}_{\infty}$. Let us consider a component $P_{\infty}$ of $\mathbb{H}^{2}-\hat{L}_{\infty}$ and a component $P_{n}$ of $\mathbb{H}^{2}-\hat{L}_{n}$ such that $\left(P_{n}\right)$ tends to $P_{\infty}$. Since $\hat{f}_{n}\left(P_{n}\right)$ converges to $\hat{f}_{\infty}\left(P_{\infty}\right)$, up to extracting a subsequence, $H_{P_{n}}^{+}$converges to a half-space $H_{P_{\infty}}^{+}$such that $\hat{f}_{\infty}\left(P_{\infty}\right) \subset \partial H_{P_{\infty}}^{+}$. Since $\hat{f}_{n}\left(\mathbb{H}^{2}\right)$ converges to $\hat{f}_{\infty}\left(\mathbb{H}^{2}\right)$, we have $\hat{f}_{\infty}\left(\mathbb{H}^{2}\right) \subset H_{P_{\infty}}^{+}$. Doing this for any component of $\mathbb{H}^{2}-L_{\infty}$, we conclude that $\hat{f}_{\infty}$ satisfies 1$)$. 
Let $\hat{x}$ be a point of $\mathbb{H}^{2}$, a support plane of $\hat{f}\left(\mathbb{H}^{2}\right)$ at $\hat{f}(\hat{x})$ is a hyperbolic plane $\Pi_{\hat{f}(\hat{x})}$ containing $\hat{f}(\hat{x})$ and such that $\hat{f}\left(\mathbb{H}^{2}\right)$ lies entirely in one of the two half-spaces bounded by $\Pi_{\hat{f}(\hat{x})}$. We will denote this half-space by $H_{\hat{f}(\hat{x})}^{+}$. Let $\hat{k} \subset \mathbb{H}^{2}$ be a closed segment, a polygonal approximation to $\hat{f}(\hat{k})$ is a finite family $\mathfrak{P}=\left\{\left(\hat{x}_{i}, \Pi_{\hat{f}\left(\hat{x}_{i}\right)}\right) \mid i=1, \ldots, p\right\}$ such that :

- the $\hat{x}_{i}$ are ordered points of $\hat{k}$;

- $\Pi_{\hat{f}\left(\hat{x}_{i}\right)}$ is a support plane at $\hat{f}\left(\hat{x}_{i}\right)$;

$-\Pi_{\hat{f}\left(\hat{x}_{i}\right)} \cap \Pi_{\hat{f}\left(\hat{x}_{i+1}\right)} \neq \emptyset$ for any $i=1, \ldots, p-1$;

- if $\hat{x}_{i-1}=\hat{x}_{i}=\hat{x}_{i+1}$, then $\Pi_{\hat{f}\left(\hat{x}_{i}\right)}$ intersects the interior of $H_{\Pi_{\hat{f}\left(\hat{x}_{i+1}\right)}^{+}}-H_{\Pi_{\hat{f}\left(\hat{x}_{i-1}\right)}^{+}}$(the planes $\Pi_{\hat{f}\left(\hat{x}_{i}\right)}$ are "ordered");

- the projection of $\Pi_{\hat{f}\left(\hat{x}_{i}\right)} \cap \Pi_{\hat{f}\left(\hat{x}_{i+1}\right)}$ to $\hat{f}\left(\mathbb{H}^{2}\right)$ intersects the subarc of $\hat{k}$ joining $\hat{x}_{i}$ to $\hat{x}_{i+1}$.

The integer $p$ is the length of the polygonal approximation. We will denote by $\theta\left(\Pi_{\hat{f}\left(\hat{x}_{i}\right)}, \Pi_{\hat{f}\left(\hat{x}_{i+1}\right)}\right)$ the internal angle of $H_{\Pi_{\hat{f}\left(\hat{x}_{i}\right)}^{+}}-H_{\Pi_{\hat{f}\left(\hat{x}_{i+1}\right)}^{+}}$.

The existence of a polygonal approximation to any arc $\hat{f}(\hat{k})$ intersecting at most once any leaf of $\hat{f}(\hat{L})$ is proved in [CEG].

The bending measure $\int_{\hat{k}} d \hat{\lambda}$ along $\hat{k}$ is defined by $\int_{\hat{k}} d \hat{\lambda}=\inf _{\mathfrak{z}} \sum_{i=1}^{p} \theta\left(\Pi_{\hat{f}\left(\hat{x}_{i}\right)}, \Pi_{\hat{f}\left(\hat{x}_{i+1}\right)}\right)$ where $\mathfrak{P}$ runs over all polygonal approximations to $\hat{f}(\hat{k})$.

It is shown in [EpM, section 1.11] that this defines a transverse measure $\lambda$ on the pleating locus of $f$.

A polygonal approximation $\mathfrak{g}=\left\{\left(\hat{x}^{i}, \Pi^{i}\right)\right\}$ to a path $f(k)$ is an $(\alpha, s)$-approximation if

$$
\text { - } \max _{1 \leq i \leq p} \theta\left(\Pi_{i}, \Pi_{i+1}\right)<\alpha \text { and }
$$

$-\max d_{\mathbb{H}^{2}}\left(\hat{x}_{i}, \hat{x}_{i+1}\right)<s$.

The existence of a $(\delta, \varepsilon)$-approximation for any $(\delta, \varepsilon)$, and any arc $\hat{k}$ is shown in EpM. In the sequel we will need to have $(\delta, \varepsilon)$-approximations with bounded length. The following lemma shows there existence.

Lemma 3.2. Let $\delta$ and $\varepsilon$ be two positive numbers such that $0<\varepsilon<\frac{\log 3}{2}$. Let $(\hat{f}, \Gamma, \rho)$ be a convex pleated surface and let $\hat{k} \subset \mathbb{H}^{2}$ be an arc that intersects the pleating locus $|\hat{\lambda}|$ transversely so that $\hat{f}(\hat{k})$ intersects at most once any leaf of $\hat{f}(|\hat{\lambda}|)$. There is a $(\delta, \varepsilon)$-approximation to $\hat{f}(\hat{k})$ with length less than $\frac{4}{\varepsilon}\left(\frac{\pi}{\delta}+1\right) l(k)+4\left(\frac{\pi}{\delta}+1\right)=B(\varepsilon, \delta, l(\hat{k}))$.

Proof. Choose $p+2$ points $\hat{x}_{0}, \ldots, \hat{x}_{p+1}$ in $\hat{k}-|\hat{\lambda}|$ such that we have $\left\{\hat{x}_{1}, \hat{x}_{p+1}\right\} \subset \partial \hat{k}, p \leq \frac{l(\hat{k})}{\varepsilon}$ and $d\left(\hat{x}_{i}, \hat{x}_{i+1}\right) \leq \varepsilon$ for any $i \in\{0, \ldots, p\}$. Choose also a support plane $\Pi_{\hat{f}\left(\hat{x}_{i}\right)}$ at $\hat{f}\left(\hat{x}_{i}\right)$ for each $i \in\{0, \ldots, p\}$. The first step of the proof will be to extend this family of support planes to obtain a polygonal approximation. There are three possible configuration for the positions of $\Pi_{\hat{f}\left(\hat{x}_{i}\right)}$ and $\Pi_{\hat{f}\left(\hat{x}_{i+1}\right)}$. Let $\hat{k}_{i}$ be the subarc of $\hat{k}$ joining $\hat{x}_{i}$ to $\hat{x}_{i+1}$.

- First configuration : $\Pi_{\hat{f}\left(\hat{x}_{i}\right)}$ intersects $\Pi_{\hat{f}\left(\hat{x}_{i+1}\right)}$ and the projection of $\Pi_{\hat{f}\left(\hat{x}_{i}\right)} \cap \Pi_{\hat{f}\left(\hat{x}_{i+1}\right)}$ to $\hat{f}\left(\mathbb{H}^{2}\right)$ intersects the arc $\hat{k}_{i} \subset \hat{k}$. In this configuration, $\left\{\left(\hat{x}_{i}, \Pi_{\hat{f}\left(\hat{x}_{i}\right)}\right) ;\left(\hat{x}_{i+1}, \Pi_{\hat{f}\left(\hat{x}_{i+1}\right)}\right)\right\}$ is already a 
polygonal approximation to $\hat{f}\left(\hat{k}_{i}\right)$.

- Second configuration : $\Pi_{\hat{f}\left(\hat{x}_{i}\right)}$ does not intersect $\Pi_{\hat{f}\left(\hat{x}_{i+1}\right)}$. Let $y$ be a point of $\hat{k}_{i}$ and let $\Pi_{\hat{f}(\hat{y})}$ be a support plane at $\hat{f}(\hat{y})$. The 3 half-spaces $H_{\hat{f}(\hat{y})}^{-}, H_{\hat{f}\left(\hat{x}_{i}\right)}^{-}$and $H_{\hat{f}\left(\hat{x}_{i+1}\right)}$ intersect the ball $b(\hat{f}(\hat{y}), \varepsilon) \subsetneq b\left(\hat{f}(\hat{y}), \frac{\log 3}{2}\right)$. By [Ga] (see also $[\underline{\mathrm{Br}}]$ ) these 3 half-spaces are not disjoint, hence $\Pi_{\hat{f}(\hat{y})}$ intersects either $\Pi_{\hat{f}\left(\hat{x}_{i}\right)}$ or $\Pi_{\hat{f}\left(\hat{x}_{i+1}\right)}$. So any support plane at a point of $\hat{f}(\hat{k})$ intersects either $\Pi_{\hat{f}\left(\hat{x}_{i}\right)}$ or $\Pi_{\hat{f}\left(\hat{x}_{i+1}\right)}$. The arc $\hat{f}\left(\hat{k}_{i}\right)$ can be extended to an arc into the set of all the support planes at $\hat{f}\left(\hat{k}_{i}\right)$. Therefore there is a point $\hat{y} \subset \operatorname{int}(\hat{k})$ and a support plane $\Pi_{\hat{f}(\hat{y})}$ at $\hat{f}(\hat{y})$ such that $\left\{\left(\hat{x}_{i}, \Pi_{\hat{f}\left(\hat{x}_{i}\right)}\right) ;\left(\hat{y}, \Pi_{\hat{f}(\hat{y})}\right) ;\left(\hat{x}_{i+1}, \Pi_{\hat{f}\left(\hat{x}_{i+1}\right)}\right)\right\}$ is a polygonal approximation to $\hat{f}\left(\hat{k}_{i}\right)$ (cf. [KeS, $\left.\S 3.4\right]$ ).

- Third configuration : $\Pi_{\hat{f}\left(\hat{x}_{i}\right)}$ intersects $\cap \Pi_{\hat{f}\left(\hat{x}_{i+1}\right)}$ but the projection of $\Pi_{\hat{f}\left(\hat{x}_{i}\right)} \cap \Pi_{\hat{f}\left(\hat{x}_{i+1}\right)}$ to $\hat{f}\left(\mathbb{H}^{2}\right)$ does not intersect $\hat{f}\left(\hat{k}_{i}\right)$. If any support plane at a point of $\hat{f}\left(\hat{k}_{i}\right)$ intersects either $\Pi_{\hat{f}\left(\hat{x}_{i}\right)}$ or $\Pi_{\hat{f}\left(\hat{x}_{i+1}\right)}$ then we can find, as in the preceding case, a point $\hat{y}$ and a support plane $\Pi_{\hat{f}(\hat{y})}$ such that $\left\{\left(\hat{x}_{i}, \Pi_{\hat{f}\left(\hat{x}_{i}\right)}\right) ;\left(\hat{y}, \Pi_{\hat{f}(\hat{y})}\right) ;\left(\hat{x}_{i+1}, \Pi_{\hat{f}\left(\hat{x}_{i+1}\right)}\right)\right\}$ is a polygonal approximation to $\hat{f}\left(\hat{k}_{i}\right)$. Otherwise, there is a point $\hat{y} \in \hat{k}$ and a support plane $\Pi_{\hat{f}(\hat{y})}$ at $\hat{f}(\hat{y})$ such that $\Pi_{\hat{f}(\hat{y})}$ does not intersect $\Pi_{\hat{f}\left(\hat{x}_{i}\right)}$ nor $\Pi_{\hat{f}\left(\hat{x}_{i+1}\right)}$. The planes $\Pi_{\hat{f}\left(\hat{x}_{i}\right)}$ and $\Pi_{\hat{f}(\hat{y})}$ are in the second configuration, so there is a point $\hat{z} \subset \hat{k}_{i}$ between $\hat{x}_{i}$ and $\hat{y}$ such that $\left\{\left(\hat{x}_{i}, \Pi_{\hat{f}\left(\hat{x}_{i}\right)}\right) ;\left(\hat{z}, \Pi_{\hat{f}(\hat{z})}\right) ;\left(\hat{y}, \Pi_{f(\hat{y})}\right)\right\}$ is a polygonal approximation to the subarc of $\hat{k}$ joining $\hat{x}_{i}$ to $\hat{y}$. Doing the same for $\Pi_{\hat{f}(\hat{y})}$ and $\Pi_{\hat{f}\left(\hat{x}_{i+1}\right)}$ we get a polygonal approximation to $\hat{f}\left(\hat{k}_{i}\right)$.

Let us do the construction above for all the components of $\hat{k}-\left\{\hat{x}_{1}, \ldots, \hat{x}_{p}\right\}$. In each component of $\hat{k}-\left\{\hat{x}_{1}, \ldots, \hat{x}_{p}\right\}$, we have added at most 3 points. So the resulting polygonal approximation has a length smaller than $4 p+4$. Let us denote by $\left\{\left(\hat{x}_{i}, \Pi_{\hat{f}\left(\hat{x}_{i}\right)}\right) / i=0, \ldots, 4 p+4\right\}$ this polygonal approximation and let us denote by $\hat{k}_{i} \subset \hat{k}$ the geodesic arc joining $\hat{x}_{i}$ to $\hat{x}_{i+1}$. We have already seen that we can extend an arc $\hat{f}\left(\hat{k}_{i}\right)$ to an arc in the set of all the support planes at $\hat{f}\left(\hat{k}_{i}\right)$. This implies that, for any $\delta$, there are points $\hat{y}_{j} \subset \hat{k}_{i}, 0 \leq j \leq q+1$ with $q \leq \frac{\theta\left(\Pi_{\hat{f}\left(\hat{x}_{i}\right)}, \Pi_{\hat{f}\left(\hat{x}_{i+1}\right)}\right)}{\delta} \leq \frac{\pi}{\delta}$, such that we have $\hat{y}_{0}=\hat{x}_{i}, \hat{y}_{q+1}=\hat{x}_{i+1}$ and $\theta\left(\Pi_{\hat{f}\left(\hat{y}_{j}\right)}, \Pi_{\hat{f}\left(\hat{y}_{j+1}\right)}\right) \leq \delta$. Choosing such points for each arc $\hat{k}_{i}, 0 \leq i \leq 4 p+3$, we get a $(\delta, \varepsilon)$-approximation with length smaller than $(4 p+4)\left(\frac{\pi}{\delta}+1\right)$. Since $p \leq \frac{l(\hat{k})}{\varepsilon}$, this polygonal approximation satisfies the conclusion of the Lemma.

The following proposition of [KeS] gives an estimate of the error which is made when approximating the bending measure :

Proposition 3.3 (KeS, Proposition 4.8). There is a universal constant $K$, and a function $s(\alpha), 0<s(\alpha)<1$, such that if $\mathfrak{A}$ is an $(\alpha, s(\alpha))$-approximation to a path $f(k)$, where $\alpha<\frac{\pi}{2}$, then we have

$$
\left|\sum_{\mathfrak{y}} \theta\left(\Pi^{i}, \Pi^{i+1}\right)-\int_{k} d \gamma\right|<K \alpha l(k)
$$

Now we will use this proposition to prove the continuity of the bending measured geodesic lamination of a converging sequence of convex pleated surfaces.

Lemma 3.4. Let $\left(\hat{f}_{n}, \Gamma_{n}, \rho_{n}\right)$ be a sequence of convex pleated surfaces converging to a pleated surface $\left(\hat{f}_{\infty}, \Gamma_{\infty}, \rho_{\infty}\right)$ and let $\hat{\lambda}_{n}$ be the bending measured geodesic lamination of $\hat{f}_{n}$. The sequence $\left(\hat{\lambda}_{n}\right)$ converges for the weak $k^{*}$ topology to a measured geodesic lamination $\hat{\lambda}_{\infty}$ and we have one of the following two situations :

- $\left(\hat{f}_{\infty}, \Gamma_{\infty}, \rho_{\infty}\right)$ is a convex pleated surface and $\hat{\lambda}_{\infty}$ is its bending measured geodesic lamination; 
- $\left(\hat{f}_{\infty}, \Gamma_{\infty}, \rho_{\infty}\right)$ is an even pleated surface, $\left|\hat{\lambda}_{\infty}\right|$ is the pleated locus of $\hat{f}_{\infty}$ and $\hat{\lambda}_{\infty}$ is obtained by endowing each leaf of $\left|\hat{\lambda}_{\infty}\right|$ with a Dirac mass with a weight equal to $\pi$.

Proof. Notice that when $\mathbb{H}^{3} / \rho_{\infty}\left(\pi_{1}(M)\right)$ is quasi-isometric to $\mathbb{H}^{3} / \rho_{n}\left(\pi_{1}(M)\right)$ this result is a consequence of results of [Bo3]. By lemma 3.1. $\left(\hat{f}_{\infty}, \Gamma_{\infty}, \rho_{\infty}\right)$ is an even or convex pleated surface. We will show that any subsequence of $\left(\hat{f}_{n}, \Gamma_{n}, \rho_{n}\right)$ contains a subsequence satisfying the conclusions of lemma 3.4, the conclusion follows.

Let us choose a subsequence such that $\left(\left|\hat{\lambda}_{n}\right|\right)$ converge in the Hausdorff topology to a geodesic lamination $\hat{L}_{\infty}$. By CEG, §5.2], the pleating locus of $\hat{f}_{\infty}$ lies in $\hat{L}_{\infty}$. Let $\hat{k} \subset \mathbb{H}^{2}$ be an arc intersecting $\hat{L}_{\infty}$ transversely such that $\hat{f}_{\infty}(\hat{k})$ intersects at most once any leaf of $\hat{f}_{\infty}\left(\hat{L}_{\infty}\right)$. Since $\left(\left|\hat{\lambda}_{n}\right|\right)$ tends to $\hat{f}\left(\hat{L}_{\infty}\right)$ in the Hausdorff topology and since $\left(\hat{f}_{n}\right)$ converge to $\hat{f}_{\infty}$, for $n$ large enough $\hat{f}_{n}(\hat{k})$ intersects at most once any leaf of $\hat{f}\left(\left|\hat{\lambda}_{n}\right|\right)$. Fix $\delta<\frac{\pi}{2}$ and $\varepsilon<s(\delta)$ and choose for each $n$ a $(\delta, \varepsilon)$-approximation $\left(\hat{x}_{i, n}, \Pi_{\hat{f}_{n}\left(\hat{x}_{i, n}\right)}\right)$ to $\hat{f}_{n}(\hat{k})$ whose length is less than the number $B(\delta, \varepsilon, l(\hat{k}))$ appearing in Lemma 3.2. Extract a subsequence such that for any $i \leq B(\delta, \varepsilon, l(\hat{k}))$, the sequence $\left(\hat{x}_{i, n}, \Pi_{\hat{f}_{n}\left(\hat{x}_{i, n}\right)}\right)$ converges and denote by $\left(\hat{x}_{i, \infty}, \Pi_{\hat{f}_{\infty}\left(\hat{x}_{i, \infty}\right)}\right)$ its limit. Thus we get a $(\delta, \varepsilon)$-approximation $\left\{\left(\hat{x}_{i, \infty}, \Pi_{\hat{f}_{\infty}\left(\hat{x}_{i, \infty}\right)}\right)\right\}$ to $\hat{f}_{\infty}(\hat{k})$ whose length is less than $B(\delta, \varepsilon, l(\hat{k}))$.

Claim 3.5. If $\left(\hat{f}_{\infty}, \Gamma_{\infty}, \rho_{\infty}\right)$ is a convex pleated surface, then $\left(\hat{\lambda}_{n}\right)$ converge to the bending measured geodesic lamination $\hat{\lambda}_{\infty}$ of $\left(\hat{f}_{\infty}, \Gamma_{\infty}, \rho_{\infty}\right)$.

Proof. Assume that $\left(\hat{f}_{\infty}, \Gamma_{\infty}, \rho_{\infty}\right)$ is a convex pleated surface and let us denote by $\hat{\lambda}_{\infty}$ its bending measured geodesic lamination. Let us recall that $\hat{\lambda}_{n}$ tends to $\hat{\lambda}_{\infty}$ if for any arc $\hat{k} \subset \mathbb{H}^{2}$ transverse to $\left|\hat{\lambda}_{\infty}\right|,\left(\int_{\hat{k}} d \hat{\lambda}_{n}\right)$ tends to $\int_{\hat{k}} d \hat{\lambda}_{\infty}$.

Let $k \subset \mathbb{H}^{2}$ be an arc transverse to $\left|\hat{\lambda}_{\infty}\right|$ and let $\left\{\left(\hat{x}_{i, n}, \Pi_{\hat{f}_{n}\left(\hat{x}_{i, n}\right)}\right)\right\}$ be the $(\delta, \varepsilon)$-approximations defined above. For any $i \leq p, \Pi_{\hat{f}_{n}\left(\hat{x}_{i, n}\right)}$ tends to $\Pi_{\hat{f}_{\infty}\left(\hat{x}_{i, \infty}\right)}$. Therefore, for $n$ large enough, we have $\sum_{i=1}^{p}\left|\theta\left(\Pi_{\hat{f}_{\infty}\left(\hat{x}_{i, \infty}\right)}, \Pi_{\hat{f}_{\infty}\left(\hat{x}_{i+1, \infty}\right)}\right)-\theta\left(\Pi_{\hat{f}_{n}\left(\hat{x}_{i, n}\right)}, \Pi_{\hat{f}_{n}\left(\hat{x}_{i+1, n}\right)}\right)\right| \leq \delta$. It follows from Proposition 3.3 that we have $\left|\sum_{i=1}^{q-1} \theta\left(\Pi_{\hat{f}_{n}\left(\hat{x}_{i, n}\right)}, \Pi_{\hat{f}_{n}\left(\hat{x}_{i+1, n}\right)}\right)-\int_{\hat{k}} d \hat{\lambda}_{n}\right|<K \delta l(\hat{k})$ for any $n \in \overline{\mathbb{N}}$. Hence we have $\left|\int_{\hat{k}} d \hat{\lambda}_{\infty}-\int_{\hat{k}} d \hat{\lambda}_{n}\right|<2 K \delta l(\hat{k})+\delta$. Letting $\delta$ tend to 0 yields $\int_{\hat{k}} d \hat{\lambda}_{n} \rightarrow \int_{\hat{k}} d \hat{\lambda}_{\infty}$. The same is true for any arc $\hat{k}$ transverse to $\left|\hat{\lambda}_{\infty}\right|$, hence $\hat{\lambda}_{n}$ converges to $\hat{\lambda}_{\infty}$.

Next we will consider the case where $\left(\hat{f}_{\infty}, \Gamma_{\infty}, \rho_{\infty}\right)$ is an even pleated surface. Let us first show that all the leaves of the pleating locus $\left|\hat{\lambda}_{\infty}\right|$ of $\left(\hat{f}_{\infty}, \Gamma_{\infty}, \rho_{\infty}\right)$ are isolated leaves, namely that their projections to $\mathbb{H}^{2} / \Gamma_{\infty}$ are isolated leaves.

Claim 3.6. When $\left(\hat{f}_{\infty}, \Gamma_{\infty}, \rho_{\infty}\right)$ is an even pleated surface, the pleating locus $\left|\hat{\lambda}_{\infty}\right|$ of $\hat{f}_{\infty}$ contains only isolated leaves.

Proof. Choose two distinct successive points $\hat{x}_{i, \infty}$ and $\hat{x}_{i+1, \infty}$ and denote by $\hat{k}_{i}$ the subarc of $\hat{k}$ joining $\hat{x}_{i, \infty}$ to $\hat{x}_{i+1, \infty}$. Let $\hat{y}$ be a point of $\operatorname{int}\left(\hat{k}_{i}\right)$ and let $\left(\Pi_{\hat{f}_{n}(\hat{y})}\right)$ be a sequence of support planes at $\hat{f}_{n}(\hat{y})$ converging to a support plane $\Pi_{\hat{f}_{\infty}(\hat{y})}$ at $\hat{f}_{\infty}(\hat{y})$. For $n$ large enough, $\hat{y}$ lies in the subarc of $\hat{k}$ joining $\hat{x}_{i, n}$ to $\hat{x}_{i+1, n}$. Since $\left\{\left(\hat{x}_{i, n}, \Pi_{\hat{f}_{n}\left(\hat{x}_{i, n}\right)}\right)\right\}$ is a $(\delta, \varepsilon)$-approximation, $\Pi_{\hat{f}_{n}(\hat{y})}$ intersects both $\Pi_{\hat{f}_{n}\left(\hat{x}_{i, n}\right)}$ and $\Pi_{\hat{f}_{n}\left(\hat{x}_{i+1, n}\right)}$ and we have $\theta\left(\Pi_{\hat{f}_{n}\left(\hat{x}_{i, n}\right)}, \Pi_{\hat{f}_{n}(\hat{y})}\right) \leq \delta$ and $\theta\left(\Pi_{\hat{f}_{n}(\hat{y})}, \Pi_{\hat{f}_{n}\left(\hat{x}_{i+1, n}\right)}\right) \leq \delta$. Letting $n$ tend to $\infty$ yields $\theta\left(\Pi_{\hat{f}_{\infty}(\hat{y})}, \Pi_{\hat{f}_{\infty}\left(\hat{x}_{i, \infty}\right)}\right) \leq \delta$ and $\theta\left(\Pi_{\hat{f}_{\infty}(\hat{y})}, \Pi_{\hat{f}_{\infty}\left(\hat{x}_{i+1, \infty}\right)}\right) \leq \delta$. Since $\left(\hat{f}_{\infty}, \Gamma_{\infty}, \rho_{\infty}\right)$ is an even pleated surface, the dihedral angle between two adjacent support planes lies in $\{0, \pi\}$. Hence we have $\theta\left(\Pi_{\hat{f}_{\infty}(\hat{y})}, \Pi_{\hat{f}_{\infty}\left(\hat{x}_{i, \infty}\right)}\right)=0$ and $\theta\left(\Pi_{\hat{f}_{\infty}(\hat{y})}, \Pi_{\hat{f}_{\infty}\left(\hat{x}_{i+1, \infty}\right)}\right)=0$. It follows that for any arc $\hat{\kappa} \subset \operatorname{int}\left(\hat{k}_{i}\right), i\left(\hat{\kappa}, \hat{\lambda}_{n}\right)$ tends to 0 . From the proof of [Bo3, Prop 27] we deduce that $\operatorname{int}\left(\hat{k}_{i}\right)$ does not intersect the pleating locus of $\hat{f}_{\infty}$. 
So we have shown that the intersection between $\hat{k}$ and the pleating locus of $\hat{f}_{\infty}$ lies in $\left\{\hat{x}_{i} \mid i=1 \ldots p\right\}$, in particular this intersection has a finite cardinal.

Endowing each leaf of $\left|\hat{\lambda}_{\infty}\right|$ with a Dirac measure whose weight is equal to $\pi$ yields a measured geodesic lamination $\hat{\lambda}_{\infty}$.

Let $r$ be a lower bound for the set $\left\{d\left(\hat{x}_{1}, \hat{x}_{2}\right) / \hat{x}_{1}\right.$ and $\hat{x}_{2}$ are two different points of $\left.\hat{k} \cap\left|\hat{\lambda}_{\infty}\right|\right\}$. By the proof of Claim [3.6. we can choose $r>0$. If $\left\{\left(\hat{x}_{i, \infty}, \Pi_{\hat{f}_{\infty}\left(\hat{x}_{i, \infty}\right)}\right)\right\}$ is a $(\delta, \varepsilon)$-approximation satisfying $\varepsilon<r$, we have $\sum_{i=1}^{q-1} \theta\left(\Pi_{\hat{f}_{\infty}\left(\hat{x}_{i, \infty}\right)}, \Pi_{\hat{f}_{\infty}\left(\hat{x}_{i+1, \infty}\right)}\right)=\int_{\hat{k}} d \hat{\lambda}_{\infty}$.

Replacing the proposition 3.3 by this equality in the proof of Claim 3.5 we get the following claim which concludes the proof of Lemma 3.4 :

Claim 3.7. The sequence $\left(\hat{\lambda}_{n}\right)$ tends to $\hat{\lambda}_{\infty}$ in $\mathscr{M} \mathscr{L}\left(\mathbb{H}^{2}\right)$.

Let us notice that in the case where $\left(\hat{f}_{\infty}, \Gamma_{\infty}, \rho_{\infty}\right)$ is even, we did not show that the projection of $\left|\hat{\lambda}_{\infty}\right|$ to $\mathbb{H} / \Gamma_{\infty}$ is compact. We will see further in the text that this is actually true.

We will now improve the description of $\left(\hat{f}_{\infty}, \Gamma_{\infty}, \rho_{\infty}\right)$ when it is an even pleated surface.

Lemma 3.8. Let $\left(\hat{f}_{n}, \Gamma_{n}, \rho_{n}\right)$ be a sequence of convex pleated surfaces converging to an even pleated surface $\left(\hat{f}_{\infty}, \Gamma_{\infty}, \rho_{\infty}\right)$. There is a surface $S$ with geodesic boundary, such that $\mathbb{H}^{2} / \Gamma_{\infty}$ is the double of $S$ and such that the pleating locus $\left|\hat{\lambda}_{\infty}\right|$ of $\hat{f}_{\infty}$ project to $\partial S \subset \mathbb{H} / \Gamma_{\infty}$. Furthermore $\partial S$ is compact.

Proof. First the following claim will define $S$.

Claim 3.9. All the connected components of $\mathbb{H}^{2}-\left|\hat{\lambda}_{\infty}\right|$ have the same image under $\hat{f}_{\infty}$.

Proof. Recall that since $\hat{f}_{\infty}$ is a pleated surface, the restriction of $\hat{f}_{\infty}$ to each connected component of $\mathbb{H}^{2}-\left|\hat{\lambda}_{\infty}\right|$ is one to one.

Assume that Claim 3.9 is not true. There exist two connected components $\hat{P}$ and $\hat{P}^{\prime}$ of $\mathbb{H}^{2}-\left|\hat{\lambda}_{\infty}\right|$ whose closures intersect and whose images under $\hat{f}_{\infty}$ are different. There is a point $\hat{y}$ lying in the boundary of the closure of $\hat{P}$ such that $f_{\infty}(\hat{y})$ lies in $\hat{f}_{\infty}\left(\hat{P}^{\prime}\right)$ or a point $\hat{y}$ lying in the boundary of the closure of $\hat{P}^{\prime}$ such that $\hat{f}_{\infty}(\hat{y})$ lies in $f_{\infty}(\hat{P})$. This two cases are similar and we will only deal with the first one. Let us denote by $\hat{l}$ the leaf of $\left|\hat{\lambda}_{\infty}\right|$ containing $\hat{y}$. By the proof of Claim [3.6. $\hat{l}$ is an isolated leaf. Let $\hat{k} \subset \mathbb{H}^{2}$ be a geodesic arc intersecting $\hat{l}$ transversally so that $\hat{k} \cap\left|\hat{\lambda}_{\infty}\right|=\{y\}$. For $n \in \overline{\mathbb{N}}$, let $\left(\hat{x}_{i, n}, \Pi_{\hat{f}_{n}\left(\hat{x}_{i, n}\right)}\right)$ be the $(\delta, \varepsilon)$-approximation to $\hat{f}_{n}(\hat{k})$ constructed at the beginning of the proof of Lemma 3.4 and let us denote by $p=B(\delta, \varepsilon, l(\hat{k}))$ its length. By Claim 3.7 the bending measure on $\hat{k}$ tends to a Dirac measure whose weight is equal to $\pi$ and whose support is $\{y\}$. It follows that there is $i$ such that $\left(\hat{x}_{i, n}\right)$ and $\left(\hat{x}_{i+1, n}\right)$ converge to $y$ and such that we have the inequality $\frac{\pi}{p} \leq \theta\left(\Pi_{\hat{f}_{n}\left(\hat{x}_{i, n}\right)}, \Pi_{f_{n}\left(\hat{x}_{i+1, n}\right)}\right) \leq \delta$ for $n$ large enough.

Let $\hat{z}$ be the point of $\hat{P}^{\prime}$ such that we have $\hat{f}_{\infty}(\hat{z})=\hat{f}_{\infty}(y)$. For any $n, \hat{z}$ lies either in a connected component $\hat{P}_{n}^{\prime}$ of $\mathbb{H}^{2}-\left|\hat{\lambda}_{n}\right|$ or in the closures of two connected components of $\mathbb{H}^{2}-\left|\hat{\lambda}_{n}\right|$, in this second case we will denote by $\hat{P}_{n}^{\prime}$ the interior of the union of these two closures. Let us extract a subsequence such that $\hat{P}_{n}$ converges to an open subsurface $\hat{P}_{\infty}^{\prime}$ of $\hat{P}^{\prime}$ (for example a subsequence such that $\left|\hat{\lambda}_{n}\right|$ converges in the Hausdorff topology). Since $\hat{f}_{n}$ converge to $\hat{f}_{\infty}$ on any compact set, the sequence $\hat{f}_{n}\left(P_{n}^{\prime}\right)$ converges to $\hat{f}_{\infty}\left(P_{\infty}^{\prime}\right) \subset \hat{f}_{\infty}\left(P^{\prime}\right)$.

The point $\hat{f}_{\infty}(\hat{y})$ lies in $\hat{f}_{\infty}\left(\hat{P}_{\infty}^{\prime}\right)$, in $\Pi_{\hat{f}_{\infty}\left(\hat{x}_{i, \infty}\right)}$ and in $\Pi_{\hat{f}_{\infty}\left(\hat{x}_{i+1, \infty}\right)}$ and we have the inequality $\frac{\pi}{p} \leq \theta\left(\Pi_{\hat{f}_{n}\left(\hat{x}_{i, n}\right)}, \Pi_{\hat{f}_{n}\left(\hat{x}_{i+1, n}\right)}\right) \leq \delta$. Therefore, for $n$ large enough, one plane among $\Pi_{\hat{f}_{n}\left(\hat{x}_{i, n}\right)}$ and $\Pi_{\hat{f}_{n}\left(\hat{x}_{i+1, n}\right)}$ intersects $\hat{f}_{n}\left(\hat{P}_{n}^{\prime}\right)$ transversely. This contradicts the convexity of $\left(\hat{f}_{n}, \Gamma_{n}, \rho_{n}\right)$ and concludes this proof. 
Next we will show that the quotient of $\mathbb{H}^{2}-\left|\hat{\lambda}_{\infty}\right|$ by $\Gamma_{\infty}$ has two connected components.

Let us extract a subsequence such that $\left(\left|\hat{\lambda}_{n}\right|\right)$ converges in the Hausdorff topology to a geodesic lamination $\hat{L}_{\infty}$ and let $\hat{x}, \hat{y} \subset \mathbb{H}^{2}-\hat{L}_{\infty}$ be two points whose images $\hat{f}_{\infty}(\hat{x})$ and $\hat{f}_{\infty}(\hat{y})$ coincides. Denote by $\hat{P}_{\hat{x}}$ and $\hat{P}_{\hat{y}}$ the connected components of $\mathbb{H}^{2}-\left|\hat{\lambda}_{\infty}\right|$ containing $\hat{x}$ and $\hat{y}$ and assume that $H_{\hat{f}_{\infty}\left(\hat{P}_{\hat{x}}\right)}^{+}$is equal to $H_{\hat{f}_{\infty}\left(\hat{P}_{\hat{y}}\right)}^{+}$. We will show the existence of an element $g_{\infty}$ of $\Gamma_{\infty}$ such that $\hat{x}=g_{\infty} \hat{y}$.

The half-spaces $H_{\hat{f}_{n}\left(\hat{P}_{\hat{x}}\right)}^{+}$and $H_{\hat{f}_{n}\left(\hat{P}_{\hat{y}}\right)}^{+}$converge to $H_{\hat{f}_{\infty}\left(\hat{P}_{\hat{x}}\right)}^{+}$and we have $\hat{f}_{n}\left(\hat{P}_{\hat{y}}\right) \subset H_{\hat{f}_{n}\left(\hat{P}_{\hat{x}}\right)}^{+}$ and $\hat{f}_{n}\left(\hat{P}_{\hat{x}}\right) \subset H_{\hat{f}_{n}\left(\hat{P}_{\hat{y}}\right)}^{+}$. It follows that the planes $\Pi_{\hat{f}_{n}(\hat{x})}$ and $\Pi_{\hat{f}_{n}(\hat{y})}$ intersect for $n$ large enough and that the sequence $\theta\left(\Pi_{\hat{f}_{n}(\hat{x})}, \Pi_{\hat{f}_{n}(\hat{y})}\right)$ tends to 0 . This implies that the distance $d_{\left(\Pi_{\hat{f}_{n}(\hat{x})} \cup \Pi_{\hat{f}_{n}(\hat{y})}\right)}\left(\hat{f}_{n}(\hat{x}), \hat{f}_{n}(\hat{y})\right)$ measured on $\Pi_{\hat{f}_{n}(\hat{x})} \cup \Pi_{\hat{f}_{n}(\hat{y})}$ tends to 0 . Since we have $d_{\left(\Pi_{\hat{f}_{n}(\hat{x})} \cup \Pi_{\hat{f}_{n}(\hat{y})}\right)}\left(\hat{f}_{n}(\hat{x}), \hat{f}_{n}(\hat{y})\right) \geq d_{\hat{f}_{n}\left(\mathbb{H}^{2}\right)}\left(\hat{f}_{n}(\hat{x}), \hat{f}_{n}(\hat{y})\right)$ and since $\hat{f}_{\infty}$ is a local homeomorphism on the complementary regions of $\left|\hat{\lambda}_{\infty}\right|$, there is, for $n$ large enough, an isometry $a_{n} \in \Gamma_{n}$ such that $d_{\mathbb{H}^{2}}\left(a_{n} \hat{y}, \hat{x}\right) \longrightarrow 0$. The distance $d_{\mathbb{H}^{2}}\left(\hat{y}, a_{n} \hat{y}\right)$ is bounded, hence, up to extracting a subsequence, $\left(a_{n}\right)$ converges to some $a_{\infty} \in \Gamma_{\infty}$. We have then $a_{\infty} \hat{y}=\hat{x}$.

This implies that any point of $\hat{f}_{n}\left(\mathbb{H}^{2}-\left|\hat{\lambda}_{\infty}\right|\right)$ has at most two preimages in $\mathbb{H}^{2} / \Gamma_{\infty}$. Combining this with Claim 3.9 we get the existence of a surface $S$ such that $\mathbb{H}^{2} / \Gamma_{\infty}$ is the double of $S$ and that the pleating locus $\left|\hat{\lambda}_{\infty}\right|$ of $\hat{f}_{\infty}$ projects to $\partial S \subset \mathbb{H}^{2} / \Gamma_{\infty}$.

It remains to show that $\partial S$ is compact. Assume the contrary, then $\partial S$ contains two asymptotic half geodesics. Let $\hat{k} \subset S$ be a geodesic arc joining these two half geodesic. The double of $\hat{k}$ is a simple closed curve $c \subset \mathbb{H}^{2} / \Gamma_{\infty}$ bounding a cusp of $\mathbb{H}^{2} / \Gamma_{\infty}$. The image of $c$ under $f_{\infty}$ is $f_{\infty}(\hat{k})$ covered twice. It follows that the curve $f_{\infty}(c)$ is homotopic to a point in $\mathbb{H}^{3} / \rho_{\infty}\left(\Gamma_{\infty}\right)$. This contradicts the assumption that the parabolic elements of $\Gamma_{\infty}$ are mapped to parabolic isometries by $\rho_{\infty}$.

We will conclude this section with two lemmas which will be used in the next sections. They are proved in [Le1] (see also [Se]). We will call them slight bending Lemmas.

Lemma 3.10. Let $(\hat{f}, \Gamma, \rho)$ be a convex pleated surface, let $\hat{x}_{1}$ and $\hat{x}_{2} \in \mathbb{H}^{2}$, let $\hat{c}_{1} \subset \mathbb{H}^{2}$ be the geodesic segment joining $\hat{x}_{1}$ to $\hat{x}_{2}$ and let $\tilde{c}_{2} \subset \mathbb{H}^{3}$ be the geodesic segment joining $\hat{f}\left(\hat{x}_{1}\right)$ to $\hat{f}\left(\hat{x}_{2}\right)$. If there exists $\varepsilon<\frac{\pi}{2}$ such that the bending measure of $\hat{f}\left(\hat{c}_{1}\right)$ is smaller than $\varepsilon$, then $\exists C_{\varepsilon}$ such that $l\left(\hat{c}_{1}\right) \leq C_{\varepsilon} l\left(\tilde{c}_{2}\right)$. Furthermore $\lim _{\varepsilon \longrightarrow 0} C_{\varepsilon}=0$ and the sum of the exterior angles that $\hat{f}\left(\hat{c}_{1}\right)$ and $\tilde{c}_{2}$ make at their vertices is smaller than $\varepsilon$.

Lemma 3.11 (Slightly bent curves are quasi-geodesics). Let $(\hat{f}, \Gamma, r)$ be a convex pleated surface, let $\hat{\lambda}$ be its bending measured geodesic lamination, let $c$ be a simple closed geodesic of $\mathbb{H}^{2} / \Gamma$ and let $c^{*}$ be the geodesic of $\mathbb{H}^{3} / r\left(\Gamma_{n}\right)$ in the homotopy class of $f(c)$. For any $\varepsilon<\frac{\pi}{2}$ there exists $C_{\varepsilon}$ and $A_{\varepsilon}$ such that if $i(c, \lambda) \leq \varepsilon$ then $l(c) \leq C_{\varepsilon}\left(l\left(c^{*}\right)+A_{\varepsilon}\right)$. Moreover $\lim _{\varepsilon \rightarrow 0} C_{\varepsilon}=1$, and $\lim _{\varepsilon \longrightarrow 0} A_{\varepsilon}=0$.

\section{The continuity of $b_{\mathscr{R}}$}

Next we will use the results of the previous section to show the continuity of $b_{\mathscr{R}}$. But first let us precise the definition of the bending measured lamination of a geometrically finite metric $\sigma \in \mathscr{G} \mathscr{F}(M)$. Let $\rho: \pi_{1}(M) \rightarrow \operatorname{Isom}\left(\mathbb{H}^{3}\right)$ be a representation associated to $\sigma$ and let $N(\rho)$ be the Nielsen core of $\rho$. There are a multi-curve $\lambda^{(p)}$ and a natural (relative to $\sigma$ ) homeomorphism $f: \partial_{\chi<0} M-\lambda^{(p)} \rightarrow \partial N(\rho)$. This homeomorphism $f$ is constructed by using the retraction map from the domain of discontinuity to the boundary of the convex core of the limit set. It is well-defined up to isotopy. The surface $\partial N(\rho)$ is the image of a convex pleated surface and therefore, it has a bending measured lamination. Let us denote by $\lambda \in \mathscr{M} \mathscr{L}(\partial M)$ the image 
under $f^{-1}$ of this bending measured lamination. Adding the leaves of $\lambda^{(p)}$ endowed with Dirac masses with weights equal to $\pi$ we get the bending measured geodesic lamination of $\sigma$. This measured lamination $\lambda$ does not depend on the choice of $\rho$ (among the representations associated to $\sigma)$.

Given a simple closed geodesic $c$ and a hyperbolic metric $s$ on $\partial_{\chi<0} M$, let $l_{s}(c)$ be the length of the corresponding $s$-geodesic. If $\gamma$ is a weighted simple closed geodesic with a weight $w(\gamma)$ we define $l_{s}(\gamma)$ by $l_{s}(\gamma)=w(\gamma) l_{s}(|\gamma|)$. This function $l_{s}$ extends continuously to a function $l_{s}: \mathscr{M} \mathscr{L}(\partial M) \rightarrow \mathbb{R}$.

Given a simple closed curve $c \in \partial_{\chi<0} M$, and a hyperbolic metric $\sigma$ on $\operatorname{int}(M)$, we denote by $c^{*}$ the closed $\sigma$-geodesic in the free homotopy class of $c$ and by $l_{\sigma}\left(c^{*}\right)$ its $\sigma$-length.

Proposition 4.1. Let $\left(\sigma_{n}\right)$ be a sequence of geometrically finite metrics on the interior of $M$ converging to a non fuchsian geometrically finite metric $\sigma_{\infty}$ and let $\lambda_{n}$ be the bending measured geodesic lamination of $\sigma_{n}$. The sequence $\dot{\lambda}_{n}$ converges to $\dot{\lambda} \in \mathscr{P}^{+}(M) / \mathscr{R}$ and the bending measured geodesic lamination of $\sigma_{\infty}$ is the representative $\lambda^{\prime}$ of $\dot{\lambda}$ that lies in $\mathscr{P}(M)$.

Proof. We are going to show that any subsequence of $\left(\sigma_{n}\right)$ contains a subsequence satisfying the conclusion of the proposition. Let us begin by a result about the curves whose length tends to 0 when $n$ tends to $\infty$.

Lemma 4.2. Let $\left(\sigma_{n}\right)$ be a sequence of geometrically finite metrics on the interior of $M$ converging algebraically and let $\left(s_{n}\right)$ be the sequence of metrics induced on $\partial M$ by a homeomorphism $h_{n}: M \rightarrow N\left(\rho_{n}\right)^{e p}$ associated to $\sigma_{n}$. Then, there is $K>0$ such that if $c \subset \partial M$ is a simple closed curves that bounds an essential disc, we have $l_{s_{n}}(c) \geq K$.

Proof. Let $D \subset M$ be an essential disc and let $c \in \partial M$ be a simple closed curve which does not bound an essential disc. We will say that $c$ intersects $\partial D$ essentially if the ends of any lift of $e$ to $\tilde{M}$ are separated by a lift of $\partial D$. As in the introduction $\mathscr{P}(M)$ contains the measured geodesic laminations satisfying conditions $a$ ), $b$ ) and $c$ ).

Claim 4.3. Let $\gamma \subset \mathscr{P}(M)$ be a weighted multi-curve and let $D \subset M$ be an essential disc; then at least one leaf of $\gamma$ intersects $\partial D$ essentially.

Proof. Let $\partial \tilde{D}$ be a lift of $\partial D$ to $\partial \tilde{M}$. This lift separates $\partial \bar{M} \approx S^{2}$ into two discs $C$ and $C^{\prime}$. Let us denote by $I_{i}, 1 \leq i \leq p$ (resp. $I_{i}^{\prime}, 1 \leq i \leq p^{\prime}$ ) the connected components of $p^{-1}(\gamma)-\partial \tilde{D}$ lying in $C$ (resp. $C^{\prime}$ ) whose endpoints lie in $\partial \tilde{D}$. These $\operatorname{arcs} I_{i}$ (resp. $I_{i}^{\prime}$ ) cut $C$ (resp. $C^{\prime}$ ) in $p+1 \operatorname{discs}$ $C_{i}, 0 \leq i \leq p$ (resp. in $p^{\prime}+1 \operatorname{discs} C_{i}^{\prime}, 0 \leq i \leq p^{\prime}$ ). The curves $\partial C_{i}$ and $\partial C_{i}^{\prime} \subset \partial \tilde{M}$ bound essential discs in $\tilde{M}$. It follows from conditions $a$ ) and $c$ ) that for any $i$, we have $\sharp\left\{\partial C_{i} \cap p^{-1}(\gamma)\right\} \geq 3$ and $\sharp\left\{\partial C_{i}^{\prime} \cap p^{-1}(\gamma)\right\} \geq 3$. If no leaf of $\gamma$ intersects $\partial D$ essentially, then, for any $i$, each point of $\partial C_{i} \cap \partial \tilde{D} \cap p^{-1}(\gamma)$ is the endpoint of an $I_{j}^{\prime}$ and each point of $\partial C_{i}^{\prime} \cap \partial \tilde{D} \cap p^{-1}(\gamma)$ is the endpoint of an $I_{j}$. This implies that $3(p+1)=2 p^{\prime}$ and that $3\left(p^{\prime}+1\right)=2 p$. We get $p+p^{\prime}=-6$. This contradiction shows that at least one leaf of $\gamma$ intersects $\partial D$ essentially.

Let $\left(c_{n}\right)$ be a sequence of simple closed curves such that each $c_{n}$ bounds an essential disc and that $l_{s_{n}}\left(c_{n}\right) \longrightarrow 0$. For large $n, c_{n}$ is the core of a wide Margulis tube $T_{n} \subset \partial M$. Let $c_{1, n}$ and $c_{2, n}$ be the boundary components of $T_{n}$ and let us choose $T_{n}$ so that we have $l_{s_{n}}\left(c_{1, n}\right)=l_{s_{n}}\left(c_{2, n}\right) \longrightarrow 0$ and $d_{s_{n}}\left(c_{i, n}, c_{n}\right) \longrightarrow \infty$. Let $\gamma \in \mathscr{P}(M)$ be a weighted multi-curve and let us extract a subsequence such that a leaf $e$ of $\gamma$ intersects all the $c_{n}$ essentially. For each $n$, let $e_{n}^{*}$ be the $\sigma_{n}$-geodesic in the homotopy class of $e$, let $\tilde{e}_{n}^{*}, \tilde{c}_{n}$ and $\tilde{T}_{n}$ be lifts of $e_{n}^{*}$, $c_{n}, T_{n}$ respectively. Let $\Pi$ be a geodesic plane containing $\tilde{e}_{n}^{*}$, let $\tilde{c}_{1, n}$ and $\tilde{c}_{2, n}$ be the components of $\partial \tilde{T}_{n}$. Let $\tilde{k}_{n}$ and $\tilde{k}_{n}^{\prime}$ be the two connected components of $\tilde{T}_{n} \cap \Pi$ and let $\tilde{\kappa}_{n}$ and $\tilde{\kappa}_{n}^{\prime}$ be the geodesic segment of $\Pi$ joining the endpoints of $\tilde{k}_{n}$ and $\tilde{k}_{n}^{\prime}$, see figure 1 . Assume that $\partial \tilde{k}_{n}$ and $\partial \tilde{k}_{n}^{\prime}$ does not lie in the preimage of $\lambda_{n}$. Let $\tilde{d}_{n}$ and $\tilde{d}_{n}^{\prime}$ be the intersections of $\Pi$ and of 

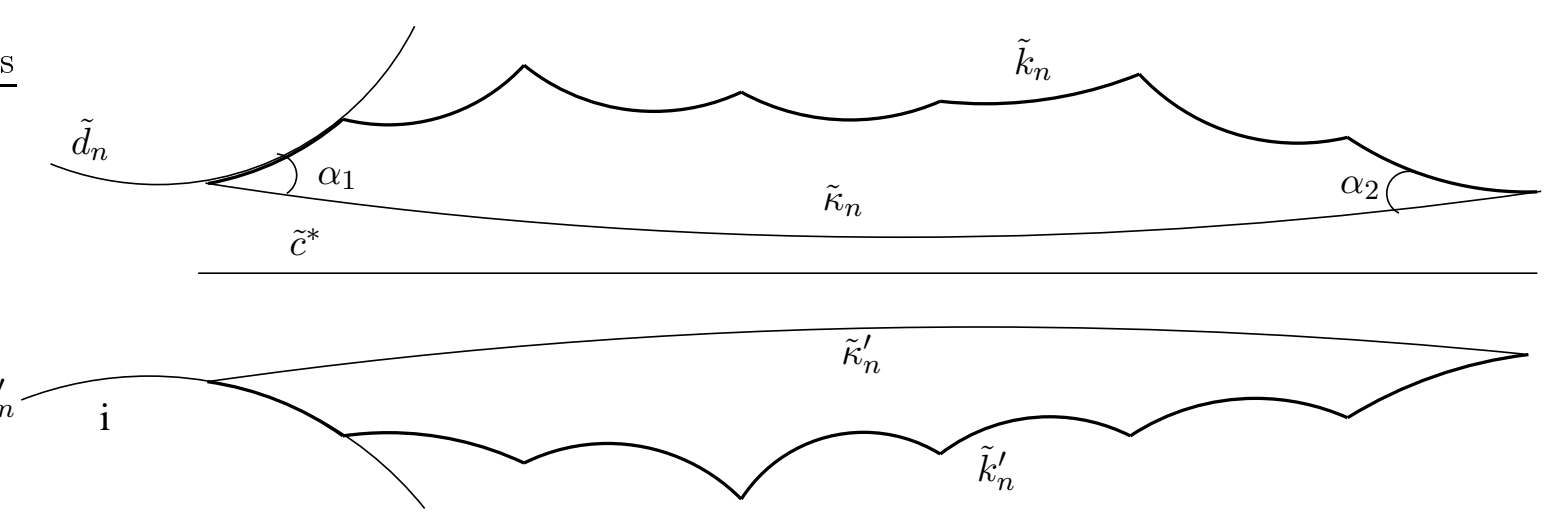

Figure 1: The section $\Pi \cap C\left(\rho_{n}\right)$

support planes at $\tilde{k}_{n} \cap \tilde{c}_{1, n}$ and at $\tilde{k}_{n}^{\prime} \cap \tilde{c}_{1, n}$ respectively. The section $C\left(\rho_{n}\right) \cap \Pi$ lies between $\tilde{d}_{n}$ and $\tilde{d}_{n}^{\prime}$. Since $\tilde{e}_{n}^{*}$ lies in $C\left(\rho_{n}\right)$, $\tilde{e}_{n}^{*}$ does not intersect $\tilde{d}_{n}$ nor $\tilde{d}_{n}^{\prime}$. The arcs $\tilde{\kappa}_{n}$ and $\tilde{\kappa}_{n}^{\prime}$ are very close, this implies that the angle $\alpha_{1}$ between $\tilde{d}_{n}$ and $\tilde{\kappa}_{n}$ is small. The same considerations apply for the angle $\alpha_{2}$ on the other vertex of $\tilde{\kappa}_{n}$. Let $\alpha<\frac{\pi}{2}$ be an upper bound for $\alpha_{1}$ and $\alpha_{2}$ and set $r_{n}=\max \left\{d\left(\tilde{x}, \tilde{\kappa}_{n}\right) / \tilde{x} \in \tilde{k}_{n}\right\} \leq \max \left\{d\left(\tilde{x}, \tilde{e}_{n}^{*}\right) / \tilde{x} \in \tilde{k}_{n}\right\}$. Any point $\tilde{x}$ lying in $\tilde{k}_{n}$ lies in a simple closed curve $\tilde{c}_{x, n} \subset \tilde{T}_{n}$ homotopic to $\tilde{c}_{n}$ such that $l_{s_{n}}\left(c_{x, n}\right) \leq l_{s_{n}}\left(c_{1, n}\right)$. This curve $\tilde{c}_{x, n}$ bounds a disc with diameter less than $l_{s_{n}}\left(c_{x, n}\right)$ which intersects $\tilde{e}_{n}^{*}$. It follows that $r_{n} \leq l_{s_{n}}\left(c_{1, n}\right) \longrightarrow 0$. A computation using Fermi coordinates (compare with [Le1, Lemme A.2]) yields $l_{\sigma_{n}}\left(\tilde{k}_{n}\right) \leq \sqrt{1+\tan ^{2} \alpha}(\operatorname{ch} r)^{2} l_{\sigma_{n}}\left(\tilde{\kappa}_{n}\right)$. Since the width of $T_{n}$ tends to infinity, we have $l_{\sigma_{n}}\left(\tilde{k}_{n}\right) \longrightarrow \infty$. So we get $l_{\sigma_{n}}\left(\tilde{\kappa}_{n}\right) \longrightarrow \infty$. Since any point of $\kappa_{n}$ is close to the geodesic $e_{n}^{*}$, we have $l_{\sigma_{n}}\left(e_{n}^{*}\right) \longrightarrow \infty$. But this contradicts the algebraic convergence of $\left(\sigma_{n}\right)$.

Since $\left(\sigma_{n}\right)$ converges to $\sigma_{\infty}$, we may choose representations $\rho_{n}: \pi_{1}(M) \rightarrow \operatorname{Isom}\left(\mathbb{H}^{3}\right)$ associated to $\sigma_{n}$ such that $\left(\rho_{n}\right)$ converges algebraically to a representation $\rho_{\infty}$ associated to $\sigma_{\infty}$ and that $\left(\rho_{n}\left(\pi_{1}(M)\right)\right)$ converges geometrically to $\rho_{\infty}\left(\pi_{1}(M)\right)$. For $n \in \overline{\mathbb{N}}$, let $\lambda_{n}$ be the bending measured geodesic lamination of $\sigma_{n}$. Denote by $\lambda_{n}^{(p)}$ the union of the leaves of $\lambda_{n}$ which have a weight equal to $\pi$. Let $M_{n}=\mathbb{H}^{3} / \rho_{n}\left(\pi_{1}(M)\right)$ and let $h_{n}: M-\lambda_{n}^{(p)} \rightarrow N\left(\rho_{n}\right)$ be a homeomorphism associated to $\sigma_{n}$.

Let $x_{\infty}$ be a point in $\partial N\left(\rho_{\infty}\right)$, let $\partial_{x_{\infty}} N\left(\rho_{\infty}\right)$ be the connected component of $\partial N\left(\rho_{\infty}\right)$ containing $x_{\infty}$ and let $S$ be a connected component of $\partial M-\lambda_{\infty}^{(p)}$ satisfying $h_{\infty}(S)=\partial_{x_{\infty}} N\left(\rho_{\infty}\right)$. Let $\tilde{x}_{\infty} \in C\left(\rho_{\infty}\right)$ be a lift of $x_{\infty}$ and let $\partial_{\tilde{x}_{\infty}} C\left(\rho_{\infty}\right)$ be the connected component of $\partial C\left(\rho_{\infty}\right)$ containing $\tilde{x}_{\infty}$. By Ta, the convex hull $C\left(\rho_{\infty}\right)$ of $L_{\rho_{\infty}}$ is the limit of $\left(C\left(\rho_{n}\right)\right)$ with respect to the Hausdorff topology. Hence there exists $\tilde{x}_{n} \subset \partial C\left(\rho_{n}\right)$ such that $\left(\tilde{x}_{n}\right)$ converges to $\tilde{x}_{\infty}$. Let us denote by $\partial_{\tilde{x}_{n}} C\left(\rho_{n}\right)$ the connected component of $\partial C\left(\rho_{n}\right)$ containing $\tilde{x}_{n}$ and by $\partial_{\tilde{x}_{n}} N\left(\rho_{n}\right)$ its projection to $N\left(\rho_{n}\right)$. There is a connected component $S_{n}$ of $\partial M-\lambda_{n}^{(p)}$ and a pleated map $g_{n}: S_{n} \rightarrow M$ onto $\partial_{\tilde{x}_{n}} C\left(\rho_{n}\right) / \rho_{n}\left(\pi_{1}(M)\right)$. Let $\left(\hat{g}_{n}, \Gamma_{n}, r_{n}\right)$ be the pleated surface that lifts $g_{n}$, namely the map $\hat{g}_{n}: \mathbb{H}^{2} \rightarrow \partial_{\tilde{x}_{n}} C\left(\rho_{n}\right)$ is onto, $r_{n}: \Gamma_{n} \rightarrow \rho_{n}\left(\pi_{1}(M)\right)$ is onto the stabilizer of $\partial_{\tilde{x}_{n}} C\left(\rho_{n}\right)$ and the quotient map $\mathbb{H}^{2} / \operatorname{ker}\left(r_{n}\right) \rightarrow \partial_{\tilde{x}_{n}} C\left(\rho_{n}\right)$ is a homeomorphism. By CEG, Theorem 5.2.2] and Lemma 4.2 a subsequence of the sequence of pleated surfaces $\left(\hat{g}_{n}, \Gamma_{n}, r_{n}\right)$ converges to a pleated surface $\left(\hat{g}_{\infty}, \Gamma_{\infty}, r_{\infty}\right)$.

By Lemma 3.1. $\left(\hat{g}_{\infty}, \Gamma_{\infty}, r_{\infty}\right)$ is a convex or even pleated surface. Since $\tilde{x}_{\infty} \in \hat{g}_{\infty}\left(\mathbb{H}^{2}\right)$, then $\hat{g}_{\infty}\left(\mathbb{H}^{2}\right) \subset \partial_{\tilde{x}_{\infty}} C\left(\rho_{\infty}\right)$ and $r_{\infty}\left(\Gamma_{\infty}\right) \subset \rho_{\infty}\left(\pi_{1}(M)\right)$ is a subgroup of the stabilizer of $\partial_{\tilde{x}_{\infty}} C\left(\rho_{\infty}\right)$.

Lemma 4.4. The quotient map $g_{\infty}: \mathbb{H}^{2} / \Gamma_{\infty} \rightarrow \partial_{x_{\infty}} N\left(\rho_{\infty}\right)$ is a homeomorphism.

Proof. Set $C_{\hat{g}_{n}}=\bigcap\left\{H_{P_{i}}^{\epsilon} / P_{i}\right.$ is a connected component of $\left.\mathbb{H}^{2}-\hat{L}\right\}$. For any $n \in \mathbb{N}$, we have $C\left(\rho_{n}\right) \subset C_{\hat{g}_{n}}$. Since $\sigma_{n}$ converges to $\sigma_{\infty}$, it follows from [Ta] that $C\left(\rho_{n}\right)$ converges to $C\left(\rho_{\infty}\right)$ in the 
Hausdorff topology. So we get $C\left(\rho_{\infty}\right) \subset C_{\hat{g}_{\infty}}$. Since $\rho_{\infty}$ is not fuchsian, we have $\operatorname{int}\left(C\left(\rho_{\infty}\right)\right) \neq \emptyset$. Therefore $\operatorname{int}\left(C_{\hat{g}_{\infty}}\right) \neq \emptyset$ and $\left(\hat{g}_{\infty}, \Gamma_{\infty}, r_{\infty}\right)$ is a convex pleated surface. By [EpM], this implies that $g_{\infty}$ is a covering map.

Let $\hat{\gamma}_{n}$ be the bending geodesic lamination of $\hat{g}_{n}$. Extract a subsequence such that $\left(\left|\hat{\gamma}_{n}\right|\right)$ converges to some geodesic lamination $\hat{L}_{\infty}$ in the Hausdorff topology.

Assume that $g_{\infty}$ is not a homeomorphism. There are two points $z \neq y \in\left(\mathbb{H}^{2}-\hat{L}_{\infty}\right) / r_{\infty}\left(\Gamma_{\infty}\right)$ such that $g_{\infty}(z)=g_{\infty}(y)$. If we lift the situation to $\mathbb{H}^{2}$, we get $\hat{z}, \hat{y} \subset \mathbb{H}^{2}-\hat{L}_{\infty}$ and $\rho_{\infty}(a) \in$ $\rho_{\infty}\left(\pi_{1}(M)\right)$ such that $\hat{z} \notin \Gamma_{\infty} \hat{y}$ and that $\hat{g}_{\infty}(\hat{z})=\rho_{\infty}(a) \circ \hat{g}_{\infty}(\hat{y})$. The sequences $\tilde{z}_{n}=\hat{g}_{n}(\hat{z})$ and $\tilde{y}_{n}=\rho_{n}(a) \circ \hat{g}_{n}(\hat{y})$ converge together to $\hat{g}_{\infty}(\hat{z})$. Let $\Pi_{\tilde{z}_{n}}$ and $\Pi_{\tilde{y}_{n}}$ be support planes of $C_{n}\left(\rho_{n}\right)$ at $\tilde{z}_{n}$ and $\tilde{y}_{n}$. The half-spaces $\left(H_{\Pi_{\tilde{z}_{n}}}^{+}\right)$and $\left(H_{\Pi_{\tilde{y}_{n}}^{+}}\right)$tend to half-spaces $H_{\hat{z}}^{+}$and $H_{\hat{y}}^{+}$respectively. These two half-spaces $H_{\hat{z}}^{+}$and $H_{\hat{y}}^{+}$are bounded by the support plane $\Pi_{\hat{g}_{\infty}(\hat{z})}$ at $\hat{g}_{\infty}(\hat{z})$. Thus we have either $H_{\tilde{z}}^{+} \cap H_{\tilde{y}}^{+}=\Pi_{\hat{g}_{\infty}(\hat{z})}$, or $H_{\tilde{z}}^{+}=H_{\tilde{y}}^{+}$.

Since $C\left(\rho_{\infty}\right) \subset C_{\hat{g}_{\infty}} \subset H_{\tilde{z}_{n}}^{+} \cap H_{\tilde{y}_{n}}^{+}$and since $\rho_{\infty}\left(\pi_{1}(M)\right)$ is not fuchsian, we have $H_{\tilde{z}}^{+} \cap H_{\tilde{y}}^{+} \neq \Pi_{\hat{g}_{\infty}(\hat{z})}$. So we have $H_{\tilde{z}}^{+}=H_{\tilde{y}}^{+}$. This implies that for large $n$, if $\Pi_{\tilde{z}_{n}}$ are $\Pi_{\tilde{y}_{n}}$ disjoint then we have either $H_{\Pi_{\tilde{z}_{n}}}^{+} \subsetneq H_{\Pi_{\tilde{y}_{n}}}^{+}$or $H_{\Pi_{\tilde{y}_{n}}}^{+} \subsetneq H_{\Pi_{\tilde{z}_{n}}}^{+}$. We get a contradiction with the fact that we have $\tilde{y}_{n} \subset C\left(\rho_{n}\right) \subset H_{\Pi_{\tilde{z}_{n}}}^{+}$and $\tilde{z}_{n} \subset C\left(\rho_{n}\right) \subset H_{\Pi_{\tilde{y}_{n}}}^{+}$. We deduce that, up extracting a subsequence, $\Pi_{\tilde{z}_{n}}$ intersects $\Pi_{\tilde{y}_{n}}$. It follows that, for $n$ large enough, $\hat{g}_{n}\left(\mathbb{H}^{2}\right)$ intersects $\rho_{n}(a) \circ \hat{g}_{n}\left(\mathbb{H}^{2}\right)$. Therefore $\hat{g}_{n}\left(\mathbb{H}^{2}\right)$ and $\rho_{n}(a) \circ \hat{g}_{n}\left(\mathbb{H}^{2}\right)$ coincide. Since $H_{\Pi_{\tilde{y}}}^{+}$is equal to $H_{\Pi_{\tilde{z}}}^{+}$, the dihedral angle $\theta\left(\Pi_{\hat{z}_{n}}, \Pi_{\hat{y}_{n}}\right)$ tends to 0 . This implies that the distance between $\tilde{z}_{n}$ and $\tilde{y}_{n}$ measured on $\hat{g}_{n}\left(\mathbb{H}^{2}\right)$ tends to 0 . Since $\hat{g}_{\infty}$ is a covering map, there is a neighborhood $\mathscr{V}(\hat{z}) \subset \mathbb{H}^{2}$ of $\hat{z}$ such that, for $n \in \overline{\mathbb{N}}$ large enough, the map $\hat{g}_{n \mid \mathscr{V}(\hat{z})}: \mathscr{V}(\hat{z}) \rightarrow \hat{g}_{n}(\mathscr{V}(\hat{z}))$ is a homeomorphism. For $n$ large enough, $\hat{y}_{n}$ lies in $g_{n}(\mathscr{V}(\hat{z}))$. Hence there is $\hat{y}_{n}^{\prime} \in \mathscr{V}(\hat{z})$ such that $\hat{g}_{n}\left(\hat{y}_{n}^{\prime}\right)=\tilde{y}_{n}$. Since $\hat{g}_{n}\left(\hat{y}_{n}^{\prime}\right)=\rho_{n}(a) \circ \hat{g}_{n}(\hat{y})$ and since $r_{n}\left(\Gamma_{n}\right)$ is the sabilizer of $\hat{g}_{n}\left(\mathbb{H}^{2}\right)$ in $\rho_{n}\left(\pi_{1}(M)\right)$, there is $a_{n} \in \Gamma_{n}$ such that $a_{n} \hat{y}=\hat{y}_{n}^{\prime}$. The point $\hat{y}_{n}^{\prime}$ lies in $\mathscr{V}(\hat{z})$, so $a_{n}$ moves $\hat{y}$ a bounded distance. Therefore there is a subsequence such that $\left(a_{n}\right)$ converge to an isometry $a_{\infty} \in \Gamma_{\infty}$. Moreover $\hat{g}_{n}\left(\hat{y}_{n}^{\prime}\right)$ tends to $\hat{g}_{\infty}(\hat{z})$, hence $\left(\hat{y}_{n}^{\prime}\right)$ tend to $\hat{z}$. Thus we get $a_{\infty} \hat{y}=\hat{z}$. This yields a contradiction with the assumption that $\hat{z} \notin \Gamma_{\infty} \hat{y}$ and concludes the proof of Lemma 4.4 .

Let $F$ be a compact subset of $S$ such that the connected components of $S-F$ are infinite annuli. Since $\Gamma_{n}$ converges geometrically to $\Gamma_{\infty}$, by Mumford Lemma (cf. [CEG]) there are $\varepsilon>0$ and maps $l_{n}: F \rightarrow \mathbb{H}^{2} / \Gamma_{n}$ with the following properties:

- $l_{n}$ is a homeomorphism onto its image;

- $l_{n}(F)$ is a connected component of the $\varepsilon$-thick part of $\mathbb{H}^{2} / \Gamma_{n}$;

- the induced representations $l_{n *}: \pi_{1}(F) \rightarrow \Gamma_{n}$ converge to an isomorphism $l_{\infty *}: \pi_{1}(F) \rightarrow \Gamma_{\infty}$;

- $g_{\infty} \circ l_{\infty}$ coincides with $h_{\infty}$ on $F \subset \partial M$.

Let us show that, for large $n, g_{n} \circ l_{n}: F \rightarrow \partial N\left(\rho_{n}\right)$ is isotopic to $h_{n}$. Let $\hat{F}$ be the universal cover of $F$ and let $\hat{l}_{n}: \hat{F} \rightarrow \mathbb{H}^{2}$ be a lift of $l_{n}$. Since $\left(l_{n *}\right)$ converges algebraically, we can choose the $\hat{l}_{n}$ such that they converge to $\hat{l}_{\infty}$ on compact sets. Since $\left(\hat{g}_{n}\right)$ converges to $\hat{g}_{\infty}$, the sequence $\left(\hat{g}_{n} \circ \hat{l}_{n}\right)$ converges to $\hat{g}_{\infty} \circ \hat{l}_{\infty}$ on compact sets.

Let $x \in \operatorname{int}(M)$. Since $\left(\sigma_{n}\right)$ converges to $\left(\sigma_{\infty}\right)$, there are diffeomorphisms $u_{n}: M \rightarrow M$ isotopic to the identity which satisfie the following : for any $k>1$ and $r>0$, there exists $n(k, r)$ such that for $n \geq n(k, r)$, the restriction of $u_{n}$ to $B(x, r) \subset\left(M, \sigma_{\infty}\right)$ is a $k$-quasi-isometry into its image in $\left(M, \sigma_{\infty}\right)$. For $n \in \overline{\mathbb{N}}$, set $M_{n}=\mathbb{H}^{3} / \rho_{n}\left(\pi_{1}(M)\right)$ and let $x_{n} \in M_{n}$ be the projection of the origin $o \in \mathbb{H}^{3}$. For $n \in \overline{\mathbb{N}}$, the metric $\sigma_{n}$ yields an identification between $\left(\operatorname{int}(M), \sigma_{n}\right)$ and $M_{n}$ such that $x$ is identified with $x_{n}$. Thus we can consider the restriction of $u_{n}$ to $\operatorname{int}(M)$ as an homeomorphism $u_{n}: M_{\infty} \rightarrow M_{n}$. 
Let $\tilde{u}_{n}: \mathbb{H}^{3} \rightarrow \mathbb{H}^{3}$ be a lift of $u_{n}$ such that $\tilde{u}_{n}(o)=o$. Since $\left(\rho_{n}\left(\pi_{1}(M)\right)\right)$ converges geometrically to $\rho_{\infty}\left(\pi_{1}(M)\right), \tilde{u}_{n}: \mathbb{H}^{3} \rightarrow \mathbb{H}^{3}$ converges to the identity on compact sets (see $\mathrm{BeP}$ ).

Since $\left(\hat{g}_{n} \circ \hat{l}_{n}\right)$ converges to $\hat{g}_{\infty} \circ \hat{l}_{\infty}$ on compact sets, the sequence $\tilde{u}_{n}^{-1} \circ \hat{g}_{n} \circ \hat{l}_{n}$ converges to $\hat{g}_{\infty} \circ \hat{l}_{\infty}$ on compact sets. Furthermore, $F$ has a compact fondamental domain. Hence $u_{n}^{-1} \circ g_{n} \circ l_{n}: F \rightarrow M_{\infty}$ converges uniformly to $g_{\infty} \circ l_{\infty}$ which coincides with $h_{\infty}$. It follows that for large $n, u_{n}^{-1} \circ g_{n} \circ l_{n}$ is isotopic to $h_{\infty \mid F}$. Therefore $g_{n} \circ l_{n}$ is isotopic to $u_{n} \circ h_{\infty \mid F}$. Since $u_{n}$ is isotopic to the identity, $u_{n} \circ h_{\infty \mid F}$ is isotopic to $h_{n}$. Thus we can change $l_{n}$ by an isotopy such that $g_{n} \circ l_{n}$ coincides with $h_{n}$ on $F$.

Let $\hat{\gamma}_{n}$ be the bending measured geodesic lamination of $\hat{g}_{n}$ and let $\gamma_{n} \in \mathscr{M} \mathscr{L}\left(\mathbb{H}^{2} / \Gamma_{n}\right)$ be its projection. Let $k \subset F$ be an arc such that $l_{\infty}(k)$ is transverse to $\left|\gamma_{\infty}\right|$ and let $\hat{k} \subset \hat{F}$ be a lift of $k$. The $\operatorname{arcs} \hat{l}_{n}(\hat{k})$ converge to $\hat{l}_{\infty}(\hat{k})$. Since the convex pleated surfaces $\left(\hat{g}_{n}, \Gamma_{n}, r_{n}\right)$ converge to $\left(\hat{g}_{\infty}, \Gamma_{\infty}, r_{\infty}\right)$, Lemma 3.4 yields $\int_{\hat{l}_{n}(\hat{k})} d \hat{\gamma}_{n} \longrightarrow \int_{\hat{l}_{\infty}(\hat{k})} d \hat{\gamma}_{\infty}$. Since, for any $n \in \overline{\mathbb{N}}, g_{n} \circ l_{n}$ coincides with $h_{n}$ on $F$, we have $\int_{k} d \lambda_{n}=\int_{\hat{l}_{n}(\hat{k})} d \hat{\gamma}_{n}$ where $\lambda_{n}$ is the bending measured geodesic lamination of $\sigma_{n}$. Thus we get $\int_{k} d \lambda_{n} \longrightarrow \int_{k} d \lambda_{\infty}$.

Doing the same for each component of $\partial M-\lambda_{\infty}^{(p)}$, we conclude that for any arc $k \subset \partial M-\lambda_{\infty}^{(p)}$ transverse to $\left|\lambda_{\infty}\right|$,we have $\int_{k} d \lambda_{n} \longrightarrow \int_{k} d \lambda_{\infty}$. It follows that $\left(\dot{\lambda}_{n}\right)$ converges in $\mathscr{M} \mathscr{L}(\partial M) / \mathscr{R}$ and that the limit $\dot{\lambda}$ differs from $\dot{\lambda}_{\infty}$ only on $\lambda^{(p)}$, namely if $\lambda$ is a representative of $|\dot{\lambda}|$ then removing from $\lambda$ the closed leaves which lie in $\lambda^{(p)}$ yields the same measured geodesic lamination as removing $\lambda^{(p)}$ from $\lambda_{\infty}$. Thus, if we show that the leaves of $\lambda^{(p)}$ are leaves of $\lambda$ with a weight greater than or equal to $\pi$, we can conclude that $\dot{\lambda}_{n}$ converges to $\dot{\lambda}_{\infty}$.

Claim 4.5. Let $\lambda$ be a representative of $\dot{\lambda}$; any leave $c$ of $\lambda^{(p)}$ is a leave of $\lambda$ and has a weight greater than or equal to $\pi$ (as a leaf of $\lambda$ ).

Proof. Let $c$ be a leaf of $\left|\lambda^{(p)}\right| \subset\left|\lambda_{\infty}\right|$. Since $\dot{\lambda}_{n}$ converges to $\dot{\lambda}$ which satisfies $|\dot{\lambda}| \subset\left|\lambda_{\infty}\right|$, then either $c$ is a leaf of $\lambda$ and we will denote its weight by $w(c)$, or $c$ is a simple closed curve disjoint from $\lambda$ and we will take $w(c)=0$.

We will prove the claim by assuming that $w(c)<\pi$ and by ending in a contradiction.

Let $S$ be a component of $\partial M-\lambda^{(p)}$ whose closure contains $c$. From the fact that $r_{n}\left(\pi_{1}(S)\right)$ converges geometrically to $r_{\infty}\left(\pi_{1}(S)\right)$ we deduce that $l_{s_{n}}(c) \longrightarrow 0$.

In the case where, up to extracting a subsequence, $i\left(c, \lambda_{n}\right)$ is equal to 0 for all $n$, it follows from [BoO, Lemma 19] that $c$ is a leaf of $\lambda$ with a weight equal to $\pi$.

Let us consider the other case and let us extract a subsequence such that $i\left(c, \lambda_{n}\right)>0$. Let $\eta>0$ be a number such that $\pi-\eta$ is less than $w(c)$. The curve $c$ lies in the boundaries of two surfaces $F^{1}$ and $F^{2}$ (which may coincide) such that the following holds : $c \subset \operatorname{int}\left(F^{1} \cup F^{2}\right)$ and either $F^{1}$ (resp. $F^{2}$ ) is a pair of pants satisfying $\operatorname{int}\left(F^{1}\right) \cap|\lambda|=\emptyset$ or there is connected component of $\lambda$ which is an arational geodesic lamination in $F^{1}$ (resp. $F^{2}$ ). We will only deal with the case where $F^{1}$ and $F^{2}$ are distinct, the other case is handled in the same way. Let $d$ be a simple closed geodesic intersecting $c$ in two points such that $i(d, \lambda-c) \leq \eta$. We have $i(c, \lambda) \leq 2 \pi-\eta$. Consider a point $x_{n}^{1}$ (respectively $x_{n}^{2}$ ) of $d \cap F^{1}-\lambda_{n}$ (respectively $d \cap F^{2}-\lambda_{n}$ ) lying in the thick part of $\left(F^{1}, s_{n}\right)$ (respectively $\left(F^{2}, s_{n}\right)$ ). We may choose the $x_{n}^{j}$ so that if $k_{n}^{1}$ and $k_{n}^{2}$ are the connected components of $d-\left\{x_{n}^{1}, x_{n}^{2}\right\}$ then we have $\int_{k_{n}^{j}} d \lambda \longrightarrow \frac{i(d, \lambda)}{2}$. The existence of such points $x_{n}^{j}$ comes from the fact that in a neighborhood $\mathscr{V}(c)$ of $c, \lambda_{n} \cap \mathscr{V}(c)$ is a family of compact arcs each one spiraling many times toward $c$ (because $c$ lies in any limit of $\lambda_{n}$ for the Hausdorff topology, see Claim 2.2) and carrying a small measure (since, by Claim 2.4 we have $\left.i\left(\lambda_{n}, c\right) \longrightarrow 0\right)$ ) Let $y_{n}^{j} \subset k_{n}^{j}$ be a point such that if $\kappa_{n}^{j}$ is the segment of $k_{n}^{j}$ joining $x_{n}^{1}$ and $y_{n}^{j}$ (cf. figure 2), then we have $\int_{\kappa_{n}^{j}} d \lambda_{n} \longrightarrow \frac{i(d, \lambda)}{4}$. Let $\kappa_{n}^{3}$ and $\kappa_{n}^{4}$ be the segments joining $x_{n}^{1}$ to $y_{n}^{2}$ and $y_{n}^{1}$ to $x_{n}^{2}$ respectively. We have $\int_{\kappa_{n}^{j}} d \lambda_{n} \longrightarrow \frac{i(c, \lambda)}{4} \leq \frac{\pi}{2}-\frac{\eta}{4}$ for any $j \in\{1,2,3,4\}$. For $j \in\{1,2,3,4\}$, consider a lift $\tilde{\kappa}_{n}^{j} \subset C\left(\rho_{n}\right) \subset \mathbb{H}^{3}$ of $\kappa_{n}^{j}$ such that $\bigcup_{j} \tilde{\kappa}_{n}^{j}$ is connected. Let $\tilde{d}_{n}^{j} \subset \mathbb{H}^{3}$ 


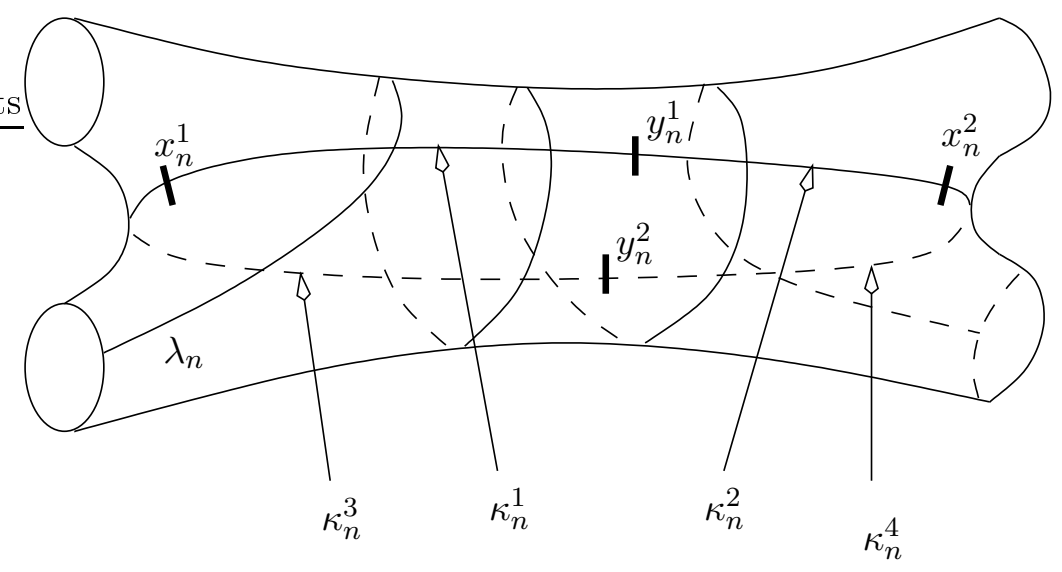

Figure 2: Cutting $c$

be the geodesic segment joining the vertices of $\tilde{\kappa}_{n}^{j}$.

If there is $j_{0}$ such that $l_{s_{n}}\left(\kappa_{n}^{j_{0}}\right)$ is bounded, then $\kappa_{n}^{j_{0}}$ is entirely contained in the thick part of $\left(F^{1} \cup F^{2}, s_{n}\right)$. Since $f_{n}\left(F^{1}\right)$ and $f_{n}\left(F^{2}\right)$ tend to pleated surfaces whose bending measured geodesic laminations are $\lambda \cap F^{j}$, we have $\lim _{n \rightarrow \infty} \int_{\kappa_{n}^{j_{0}}} d \lambda_{n} \leq i(d, \lambda-c) \leq \eta$. Furthermore, we have $\int_{\kappa_{n}^{j_{0}}} d \lambda_{n} \longrightarrow \frac{i(c, \lambda)}{4}$, hence $i(c, \lambda) \leq 4 \eta$. Since $d$ intersects $c$ transversally, and since $l_{s_{n}}(d) \longrightarrow 0, l_{s_{n}}(d)$ tends to $\infty$. Taking $\eta<\frac{\pi}{8}$, we get from the slight bending lemmas (Lemma 3.11) that $l_{\sigma_{n}}\left(d^{*}\right) \geq C_{4 \eta}\left(l_{s_{n}}(d)+A_{4 \eta}\right)$. Thus $l_{\sigma_{n}}\left(d^{*}\right)$ tends to $\infty$ contradicting the algebraic convergence of $\rho_{n}$.

Thus we have $\forall j, l_{s_{n}}\left(\kappa_{n}^{j}\right) \longrightarrow \infty$. The slight bending Lemmas (Lemma 3.10) says that there is $C>0$ such that $l_{s_{n}}\left(\tilde{\kappa}_{n}^{j}\right) \leq C l_{\sigma_{n}}\left(\tilde{d}_{n}^{j}\right)$ and that the interior angle between two adjacent segments $\tilde{d}_{n}^{j}$ is greater than $\frac{\eta}{2}$. Let $a$ be the element of $\pi_{1}(M)$ such that $\rho_{n}(a)$ fixes $d^{*}$ and let $\tilde{d}_{n}=\bigcup_{i \in \mathbb{Z} ; j=1,2,3,4} a^{i}\left(\kappa_{n}^{j}\right)$. The curve $\tilde{d}_{n}$ is the union of long geodesic segments with interior angles greater than $\frac{\eta}{2}$. It follows from a classical result (see [Ot2] for example) that there is $K$ such that $l\left(\tilde{d}_{n} / a\right)$ is smaller than $K l_{\sigma_{n}}\left(d^{*}\right)$. Thus we get $l_{s_{n}}(d) \leq C l\left(\tilde{d}_{n} / a\right) \leq C K l_{\sigma_{n}}\left(d^{*}\right)$. Since $l_{s_{n}}(d) \longrightarrow \infty$, this yields a contradiction with the algebraic convergence of $\sigma_{n}$.

It follows that $\dot{\lambda}=\dot{\lambda}_{\infty}$. Thus Proposition 4.1 is proved.

\section{References}

[AnC] J.W. Anderson, R.D. Canary, Algebraic limits of Kleinian groups which rearrange the pages of a book, Invent. Math. 126 (1996), 205-214.

[BeP] R. Benedetti, C. Petronio, Lectures on hyperbolic geometry, (1992).

[Bo1] F. Bonahon, Bouts des variétés hyperboliques de dimension 3, Ann. of Math. (2) 124 (1986), 71-158.

[Bo2] F. Bonahon, Variations of the boundary of 3-dimensionnal hyperbolic convex cores, J. Diff. Geom. 50 (1998), 1-24.

[Bo3] F. Bonahon, Shearing hyperbolic surfaces, bending pleated surfaces and Thurston's symplectic form, Ann. Fac. Sci. Toulouse Math. 5 (1996), 233-297.

[BoO] F. Bonahon, J.-P. Otal, Laminations mesurées de plissage des variétés hyperboliques de dimension 3, Prépublication de l'UMPA n ${ }^{\circ} 285$ (2001).

[Br] M. Bridgeman, Average bending of convex pleated planes in hyperbolic three-space, Invent. Math. 132 (1998), 381-391. 
[CEG] R.D. Canary, D.B.A. Epstein, P. Green, Notes on notes of Thurston, Analytical and Geometrical Aspects of hyperbolic Space (1987), 3-92.

[EpM] D.B.A. Epstein, A.Marden, Convex hulls in hyperbolic space, a theorem of Sullivan, and measured pleated surfaces, Analytical and Geometric Aspects of Hyperbolic Space (1987), 113-253.

[Ga] D. Gabai, On the geometric and topological rigidity of hyperbolic 3-manifolds, J. Amer. Math. Soc. 10 (1997), 37-74.

[Jor] T. Jørgensen, On discrete groups of Möbius transformations, Amer. J. Math. 98 (1976), 739-749.

[KaT] Y. Kamishima, S. P. Tan, Deformation spaces on geometric structures, Aspects of low-dimensional manifolds, Adv. Stud. Pure Math. 20 (1992), 263-299.

[KeS] L. Keen, C. Series, Continuity of convex hull boundaries, Pac. J. Math. 127 (1988), 457-519.

[Le1] C. Lecuire, Plissage des variété hyperboliques de dimension 3, prépublication de l'UMPA nº 301 (2002).

[Le2] C. Lecuire, Bending map and strong convergence, preprint.

[Ot1] J.-P. Otal, Sur la dégénérescence des groupes de Schottky, Duke Math. J. 74 (1994), 777-792.

[Ot2] J.-P. Otal, Le théorème d'hyperbolisation pour les variétés fibrées de dimension 3, Astérisque 235 (1996).

[Se] C. Series, Quasifuchsian groups with small bending, Warwick preprint 2002.

[Ta] E. Taylor, Geometric finitness and the convergence of Kleinian groups, Com. Anal. Geom. 5 (1997), 497-533.

[Th] W.P. Thurston, The topology and geometry of 3-manifolds, Notes de cours, Université de Princeton, 1976-79. 